\title{
Implementation of visual languages using pattern-based specifications
}

\author{
Carsten Schmidt and Uwe Kastens*,† \\ University of Paderborn, Fürstenallee 11, 33102 Paderborn, Germany
}

\begin{abstract}
SUMMARY
The implementation of visual languages requires a wide range of conceptual and technical knowledge from issues of user interface design and graphical implementation to aspects of analysis and transformation for languages in general. We present a powerful toolset that incorporates such knowledge. Our toolset generates editors from high-level specifications. A language is specified by identifying certain patterns in the language structure and selecting a visual representation from a set of precoined solutions. Visual programs are represented by attributed abstract trees. Therefore, further phases of processing visual programs can be generated by state-of-the-art tools for language implementation. We demonstrate that even challenging visual languages can be implemented with reasonably little effort and with rather limited technical knowledge. The approach is suitable for a large variety of visual language styles. Copyright (c) 2003 John Wiley \& Sons, Ltd.
\end{abstract}

KEY WORDS: visual programming; graphical environments; visual patterns; reusable libraries

\section{INTRODUCTION}

Visual languages have an important role in modeling systems, specification of software, and in specific application domains. By using visual properties like spatial placement or line connections complex structures can be presented so that humans can understand them quickly. Visual languages can be based on domain-specific metaphors, so that domain specialists can use their conventional way of description and abstraction. Two examples of successful visual languages are UML [1] and LabVIEW [2]. The Unified Modeling Language (UML) is used to model requirements, design and implementation of object-oriented software systems. LabVIEW is a dataflow language, that allows the implementation of measurement and control systems. One reason for its success is its domain-specific visual metaphor of 'circuit diagrams'.

*Correspondence to: Uwe Kastens, University of Paderborn, Fürstenallee 11, 33102 Paderborn, Germany.

${ }^{\dagger}$ E-mail: uwe@uni-paderborn.de 


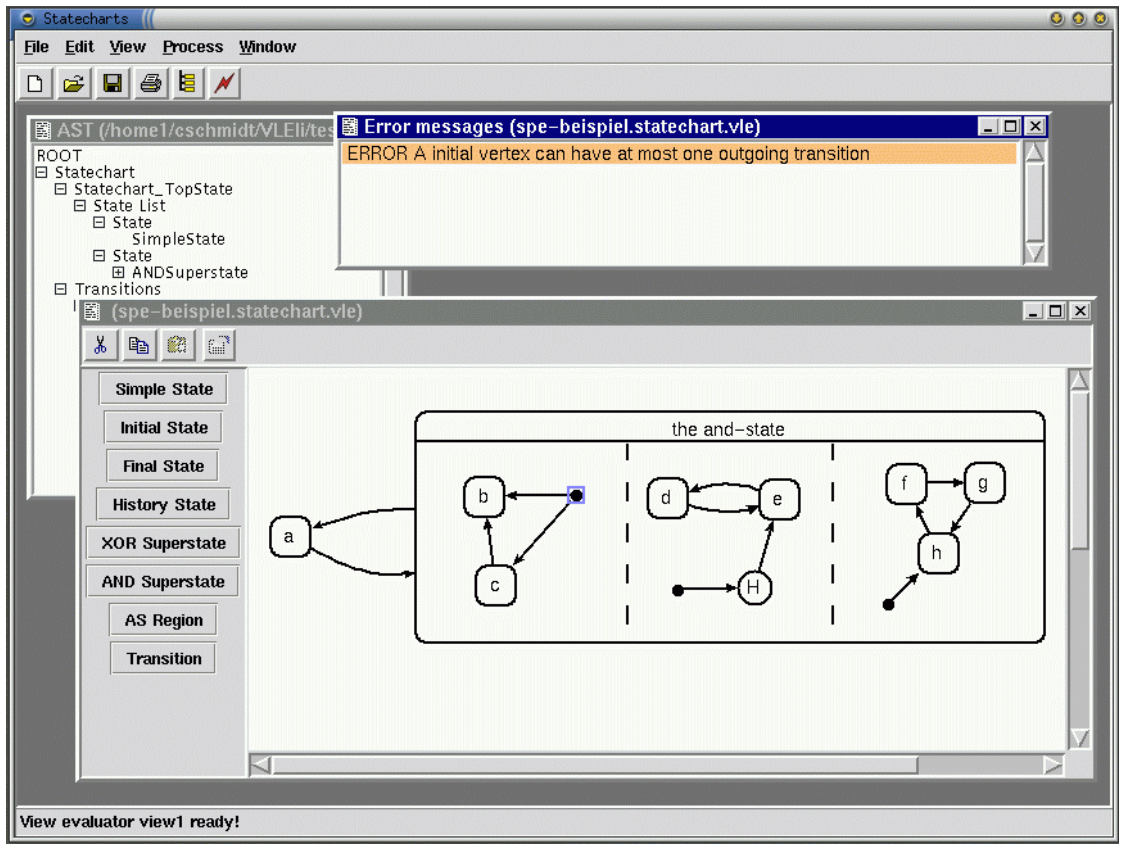

Figure 1. A structure editor for Statecharts.

For working with a visual language, a specialized graphical frontend is needed. In contrast to textual languages, general purpose editors are insufficient for visual languages because each visual language has its particular graphical requirements. The frontend should provide methods to aid efficient drawing and restructuring of visual expressions. Often, language-specific structure editors are used as frontends for visual languages. The visual program is stored in a language-dependent data structure. The user interacts with one or more visual representations. Edit operations are directly applied to the underlying structure and after a change the graphical representation is recomputed.

This article presents a new method and new tools for the implementation of visual languages. Our system VL-Eli [3] generates a structure editor as graphical frontend from a high-level specification. Since our approach is based on tree grammars and definition tables, we can use tools for further processing, that have been originally designed for processing textual languages. We use the Eli system [4-6] which contains tools and modules for a wide range of language processing tasks.

Throughout this article we use the Statechart language in the visual notation of UML [1] as a running example. Figure 1 shows a screenshot of the visual structure editor our tool has generated for that language. The screenshot shows three different views on the Statechart structure. There is a graphical view, an abstract structure tree view, and an error view. The Statechart consists mainly of an AND-superstate. It has three regions containing several simple states and transitions each. 


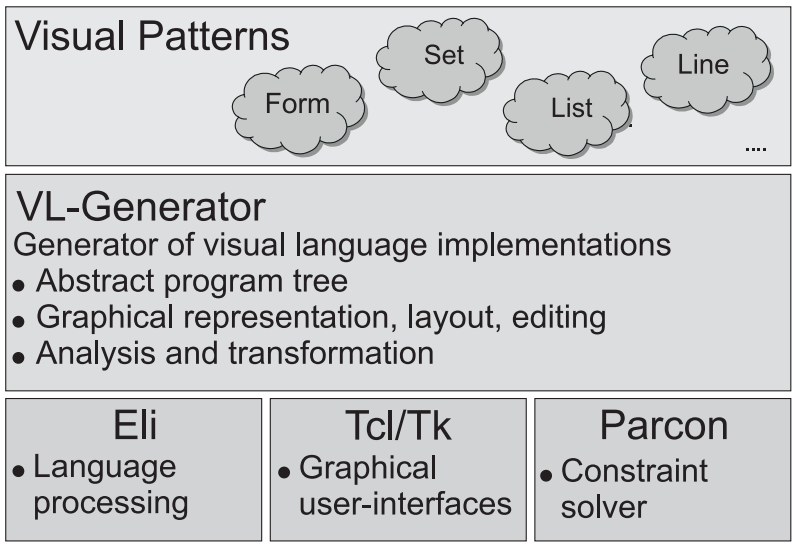

Figure 2. The VL-Eli system.

The editor supports direct manipulation: the user can drag new language elements from the left side of the graphical view and drop them where appropriate. The elements can be selected and moved or deleted as desired. Furthermore, specialized layout mechanisms are provided. For example, a constraint-solver is applied to adjust sizes and positions of the states, so that non-overlapping and correct containment of states is warranted.

The specification of the graphical properties of a visual editor is mainly based on the concept of visual patterns. The language developer identifies certain patterns in the visual language and selects a precoined solution. For example, states in UML Statechart diagrams are arranged according to the Set pattern, i.e. they have the role of a set element. They are an unordered collection of elements, which can be placed at arbitrary positions in a two-dimensional region. The language developer selects a precoined specification module for Set representations and parameterizes the details of the representation by a variety of options. All mentioned graphical and interactive properties like edit operations or layout adjustments are provided by that specification module. The language developer does not even have to know that a constraint solver is used for the layout. Most of the frontend implementation can be done by applying such visual patterns. Unusual graphical representations can be integrated by hand-coded specifications.

Figure 2 gives an overview of our system. The VL-Generator generates visual structure editors from specifications. It is built on top of tools for graphical support (Tcl/Tk [7] and Parcon [8]) and for language implementation in general (Eli [6]). The topmost layer contains variants of visual patterns, each of which encapsulates the implementation of visual language elements in terms of specifications for the VL-Generator.

The VL-Generator [9] is conceptually based on attribute grammars [10]. It generates visual editors which operate on abstract program trees as their central data structures. They perform computations during tree walks as specified for the particular language. The computations create and modify the graphical representation of the program via the interface to the graphical support tools taken from 
the bottom layer. The provided interface to Tcl/Tk allows one to arrange graphical objects, associate them with their tree context and define interactive behavior. The interface to Parcon allows one to define constraint variables accessible in tree computations, compute constraint networks and initiate the constraint solver. Both interfaces translate 'complete descriptions' (graphical representations or constraint networks, respectively) into deltas from the previous state, which are transmitted to the tools.

The abstract program tree and the attribute grammar method enable the direct connection to many tools in the Eli system, which solve a wide range of general language implementation tasks $[5,6]$. The specification modules in the Eli system, e.g. for name or type analysis, encapsulate predefined attribute computations, which can be applied by symbol inheritance. Furthermore, Eli provides generators, module libraries, and library functions. Generators automatically process specification files of dedicated types. The generated products are either automatically included in the framework or they are used in other parts of the specification. Module libraries can influence generators or provide precoined specifications. Frequently used generators are PDL (property definition language) and PTG (pattern-based text generator), which generate functionality to store and retrieve object properties and to generate structured output text, respectively [4]. Module libraries provide commonly needed functions, e.g. for string storage or for bindings in environments. Thus, Eli provides the tools necessary for program analysis and code generation. The most important tool for visual language implementation-attribute grammars-is described in Section 2.

The topmost layer contains a set of reusable descriptions of various graphical language elements in terms of the underlying layers. They are used to compose a visual language implementation without writing lower level specifications explicitly. Each such description is considered to be a variant of an abstraction that we call a visual pattern. It comprises the common properties of visual language elements with respect to their abstract structure, the interaction operations needed for them, and their visual concept [11]. For example, the List pattern represents an ordered sequence, allows element insertion and deletion, and visualizes the structure by drawing the elements side by side in a row. A language designer instantiates such a pattern variant and associates its components with certain constructs of the tree grammar. This concept of visual patterns, as well as the concrete implementation variants and the applicability for different language styles is described in Section 3.

We began the development of our tools with a field study of visual languages. We used the studied languages continuously to validate our approach and the software. In Section 4 we present experiences that we gained during that process. We discuss the quality of the user interface and the response time of generated editors. Furthermore, we evaluate the quantitative and qualitative characteristics of the languages and the specifications. Conclusions for future developments are drawn from the lessons learned.

There are several other tools which generate visual structure editors, e.g. VPE [12], GenGed [13], DiaGen [14] Progres [15] and LOGGIE [16]. Their functionality is comparable to that of the middle layer of our approach. However, only a few systems like that of Crimi et al. [17] try to support general language processing tasks, or provide a more abstract specification level, e.g. Costagliola et al. $[18,19]$. In particular, the huge number of tree-based tools for common language implementation tasks (e.g. unparser generators for textual representations) cannot be integrated directly. With our approach we do not aim at the functionality of visual editors that recognize the structure of drawings by visual parsing methods, e.g. [14,19-21]. Section 5 discusses the work related to our approach in detail. 


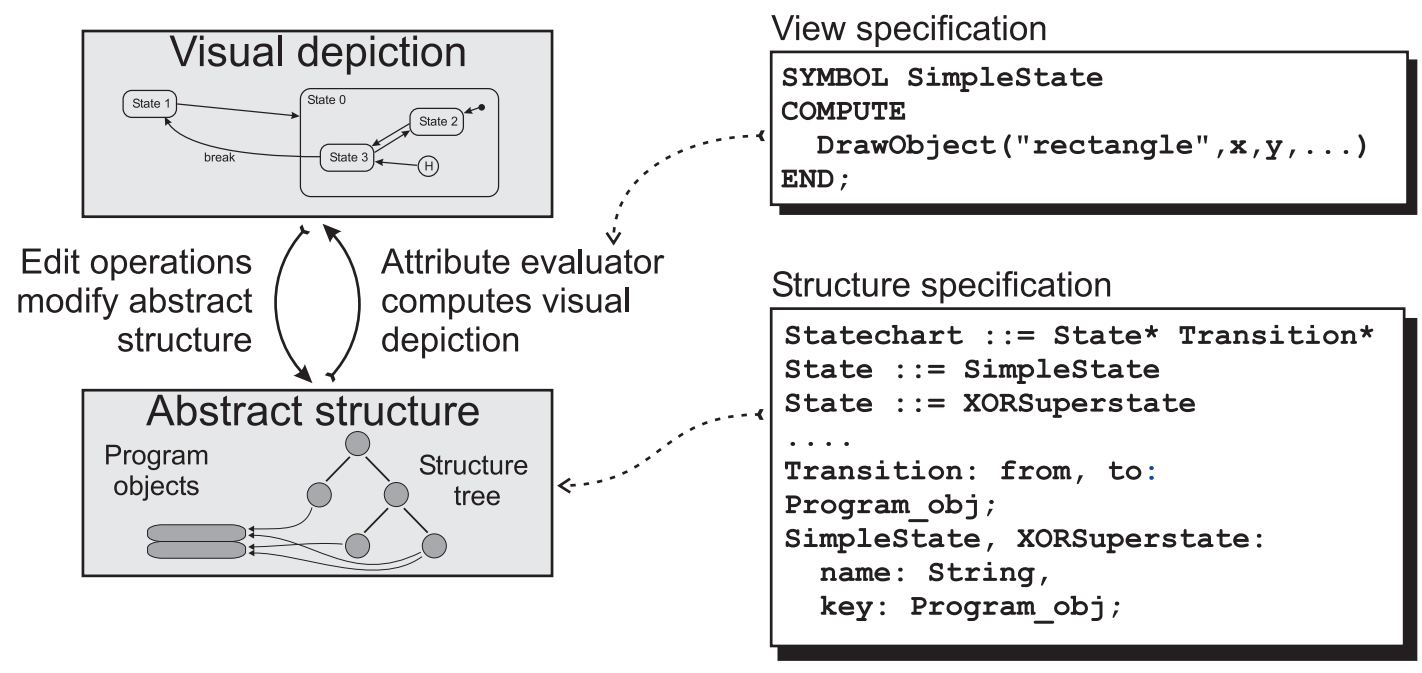

Figure 3. Concept of the VL-Generator.

\section{THE VL-GENERATOR}

Our approach for generating visual structure editors is based on the attribute grammar method (Figure 3). Attributed trees are the central data structure used to represent visual programs. They are specified by a context-free grammar, the tree grammar. Computations that visualize program constructs are associated to tree contexts (e.g. the Simplestate context). They use and yield attribute values of tree nodes, and thus propagate information through the tree. Such computations are specified by an attribute grammar. A generator analyzes the dependencies between the computations, schedules a tree walk that is suitable for any tree, and implements it by an attribute evaluator. During attribute evaluation, the graphical representation is drawn into the graphical workspace. The user can interact with the visual representation. Some of the operations cause modifications of the tree (insertion or deletion of subtrees) or of the attached data. Such modifications initiate an update of the graphical representation.

Attribute grammar specifications allow powerful abstractions, modularization, and reuse of component specifications. We use these facilities for our method of reusable visual patterns. Abstract program trees and tree walking analysis and transformation are standard techniques in compiler construction. Hence, tools for tasks beyond program editing like type analysis or code generation can be directly combined with our approach for generating visual structure editors.

In this section we explain the characteristic aspects of the method of computations in trees along with our example language Statecharts. Deeper information on that method can be obtained from attribute grammar literature [22], compiler text books, like [23], and tool descriptions, such as [24,25]. In the 


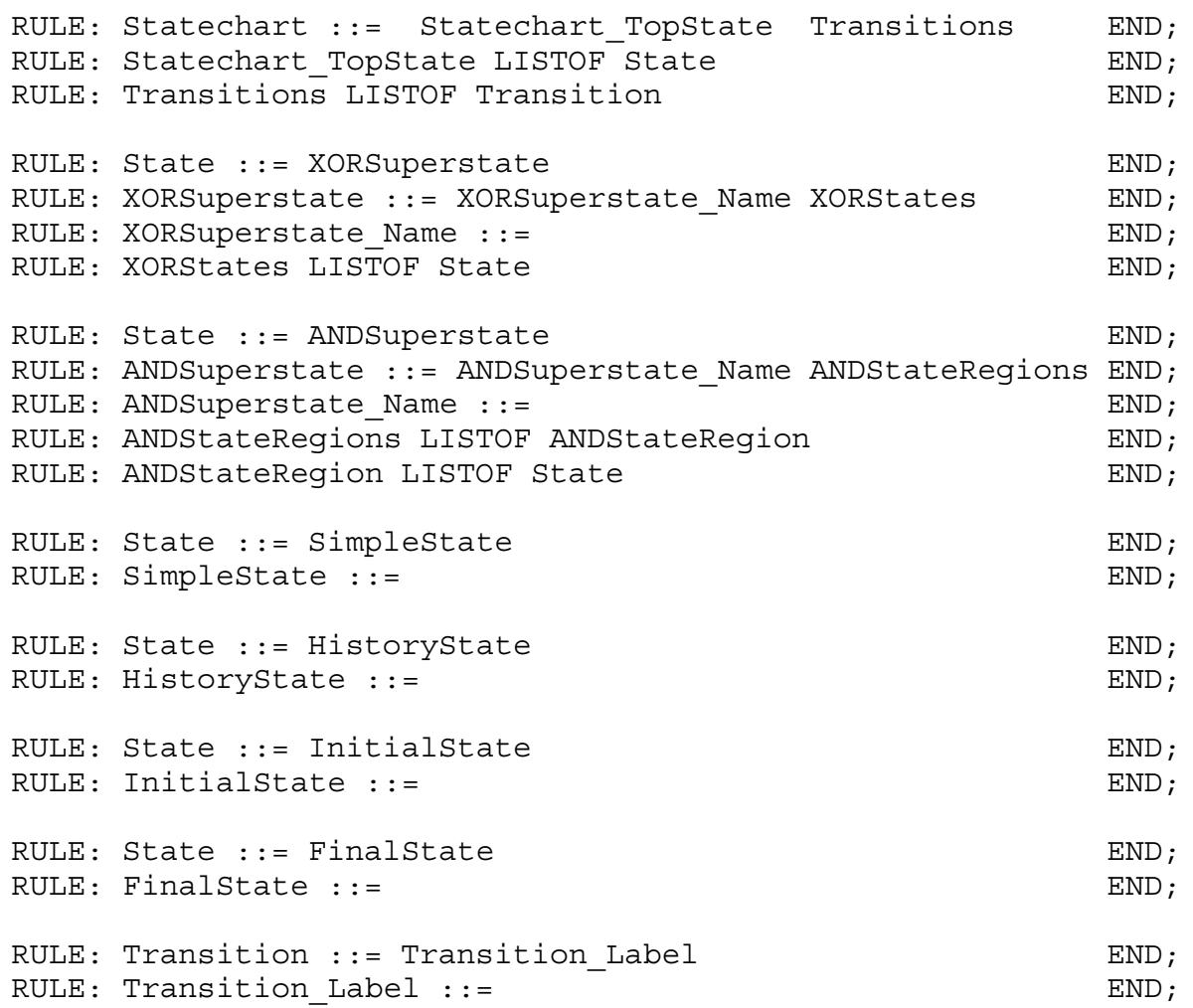

Figure 4. A tree grammar for Statecharts.

second subsection we detail the layout mechanisms provided by the VL-Generator. Last but not least the definition of cross-relations and semantic constraints is described.

\subsection{Computations in attributed trees}

The context-free grammar shown in Figure 4 is the tree grammar which specifies trees to represent the abstract structure of a Statechart. It consists of arbitrary numbers of states and transitions. There are six kinds of states. The two kinds of superstates contain states recursively. All transitions are represented on the top level of the trees because they may connect states which belong to different parts of the tree. The grammar notation uses a special form of productions to describe a sequence of subtrees: LISTOF State stands for an arbitrary number of State subtrees.

In addition to the shown grammar, persistent attributes are associated to tree symbols. For example, states have an attribute name. There is a special type of attribute to represent non-tree relations. The nodes that are connected to each other have a reference to the same connection object. In the 


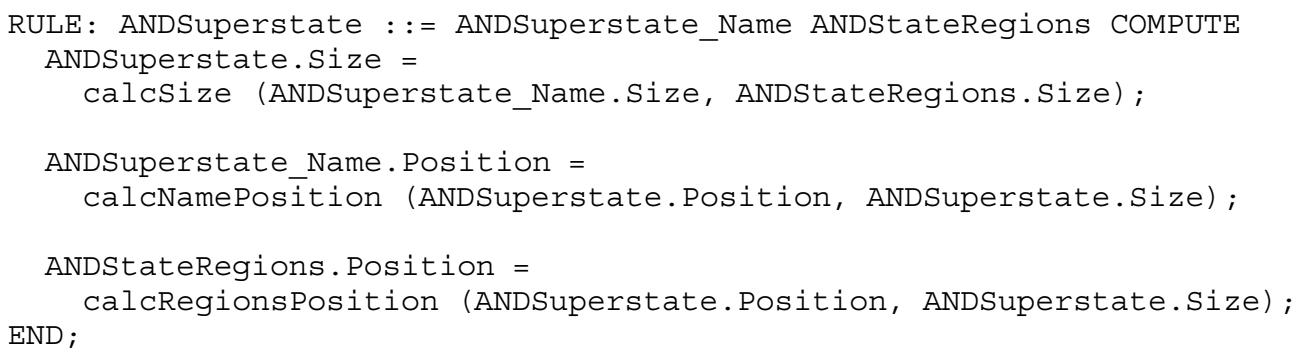

Figure 5. Layout computations for ANDSuperstates.

case of our Statecharts a state node and a line node are connected in that way. More details about cross references are given in Section 2.3.

The tree grammar is the skeleton of an attribute grammar which specifies computations to be executed on the tree. Computations are formulated in terms of the rule context in which they are to be executed. They use attribute values of the symbols in that context and may yield an attribute value as a result. An example is given in Figure 5. In general, a language specification consists of one tree grammar specification (like that in Figure 4) and multiple modules of attribute computations (as in Figure 5). Each module is responsible for a specific task, e.g. checking for semantical correctness, computing graphical representations, or translating the visual program to a target representation.

We demonstrate the principle of attribute grammars by a simplified specification of the computation of layout information (Figure 5). For each tree node that represents a visual component two attributes are computed: the Size attribute characterizes the minimal requirements (width, height, etc.) for the graphical representation of the subtree. The Position attribute defines where to draw that subtree.

The rule context that describes the structure of an ANDSuperstate has three computations. The Size attribute of the whole construct is computed by a function call which combines the Size requirements of the two subtrees. The other two computations determine the Position attributes of the subtrees using the Position of the whole construct and the Size information. (We do not discuss the internals of the functions here. They apply specific operations to realize the desired layout effects.)

Similar computational structures are specified in other rule contexts. The size information is combined upward through the tree, the position information is decided and refined downward. The specification fragment in Figure 6 demonstrates the computation of those values for ANDStateRegion nodes. Constructs for remote attribute access, CONSTITUENTS and INCLUDING, are used to propagate the information through the tree structure that connects an ANDStateRegions node with its elements. The construct

CONSTITUENTS ANDStateRegion.Size WITH (...)

accesses the Size attribute of all ANDStateRegion nodes in the subtree and combines them using the functions specified in the WITH clause. The notation

INCLUDING ANDStateRegions.Position 


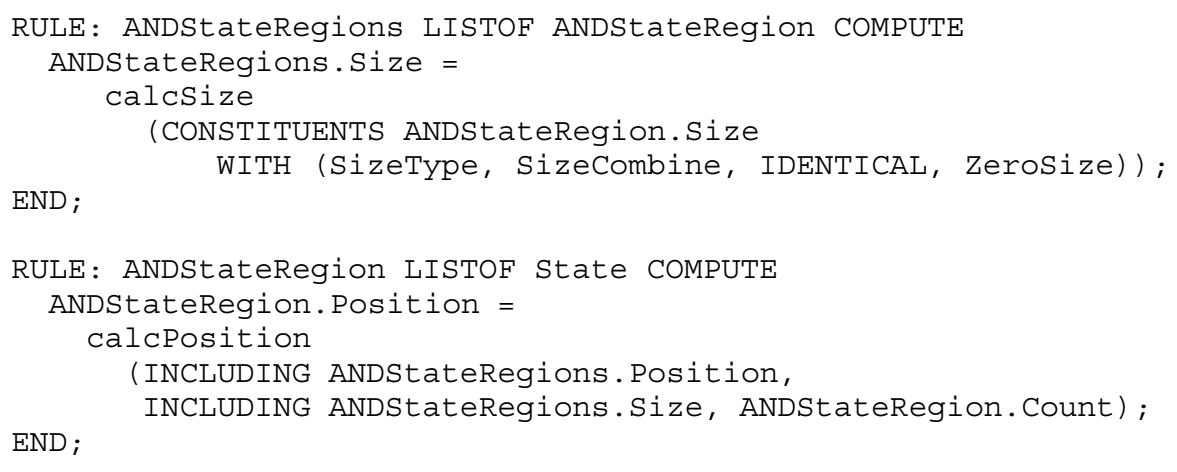

Figure 6. Layout computation for ANDStateRegions.

in the second rule refers to the Position attribute of the next node of type ANDStateRegions above the current context. These remote access constructs are useful to propagate information up or down through the tree without explicitly referring to the rule contexts in between.

The notation of attribute grammar specifications, as shown for the layout computations above, is not suitable for the reuse of specifications. Even if the computational pattern is the same, the computations have to be repeated for each rule context with suitable names and numbers of symbols. However, the specification language of the attribute evaluator generator provides powerful abstraction mechanisms: symbol computation and inheritance of computational roles [26]. Together with the constructs of remote attribute access they provide the notational basis for reusable specifications of visual patterns as described in this article.

The concept of symbol computations allows one to abstract from specific rule contexts. The associated computations are to be executed for every occurrence of the symbol in the tree. The fragment in Figure 7 reformulates the Size and Position computations shown above in terms of symbol computations.

The example in Figure 7 specifies layout computations for two related symbol contexts, the sequence ANDStateRegions and its elements ANDStateRegion. In fact, this is a more general computational pattern for size and position information in linear sequences of subtrees. The specification language allows to abstract from the specific grammar symbols and to associate the computations to abstract class symbols (Figure 8). In this case they represent the pair of the roles List and ListElement.

Such abstract specifications of computational roles can be inherited by pairs of concrete grammar symbols. The effect of the rule computations described above is then achieved by

$\begin{array}{llll}\text { SYMBOL ANDStateRegions INHERITS List } & \text { END; } \\ \text { SYMBOL ANDStateRegion INHERITS ListElement END; }\end{array}$

If there was another sequence structure in our language whose elements were to be displayed side by side in a row, its symbols could inherit the same computations. Examples for such reuse are shown in later sections. 


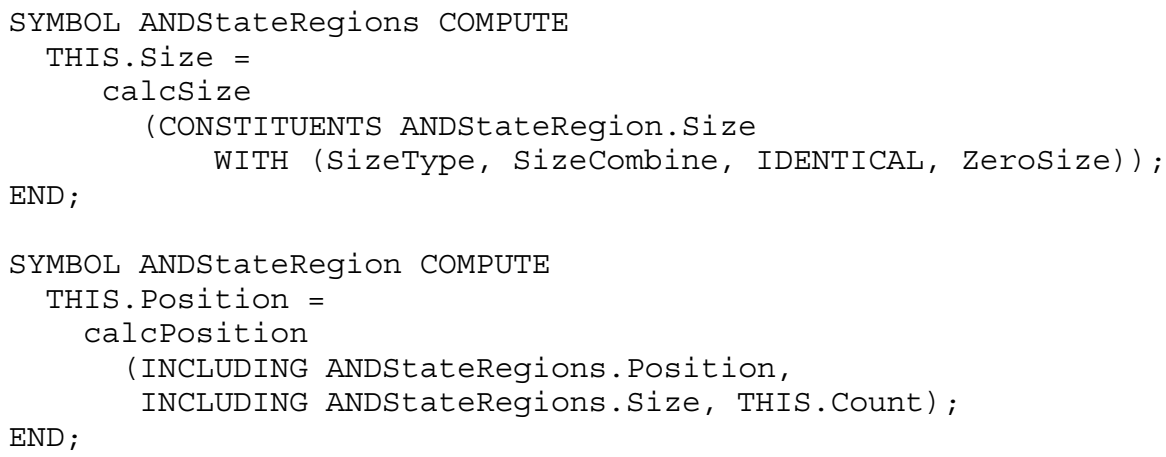

Figure 7. Layout computation in terms of symbol computations.

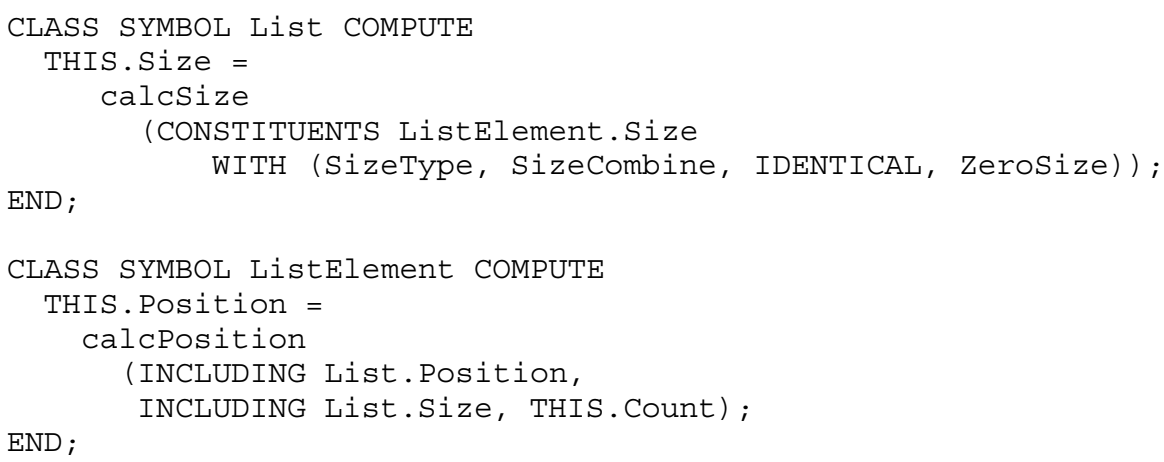

Figure 8. Symbol computations for the List pattern.

Note, that the List pattern encapsulates a particular computation of a graphical representation whereas the LISTOF constructs specifies the underlying grammar structure. Lists in the underlying grammar could also be represented by other visual patterns, e.g. by the Set or Stack pattern (compare Table I).

The combination of the notational concepts symbol computation, remote attribute access, and inheritance of computational roles supports the development of reusable specifications. Systematic solutions of tasks like layout computations can be specified completely independent of particular tree grammar productions. That allows one to separate the development of the specification. Specialists provide a library of computational roles, say for layout computation, and visual language developers decide which grammar symbol inherits which role of the library. The visual patterns, one of the central aspects of our approach, as well as name and type analysis modules in the Eli [26] are based on this principle. 
When a language developer applies computational roles of a library, certain conditions have to be fulfilled. For example, when the ListElement role is inherited, the developer must ensure, that a node with the role List is a parent in all valid trees. These nodes are an important aspect of a visual pattern, since they reflect the structure of the presented information. Validity of a pattern application is automatically checked when the attribute evaluator is generated.

\subsection{Support of layout computations}

In general, the implementation of sufficient layout mechanisms is a challenging task. The problem is to find sufficient values of graphical attributes like sizes or positions, such that (1) the representation meets the language constraints (e.g. correct containment, alignment, non-overlapping), and (2) the representation is clear and understandable according to general perception and user intentions [27].

There are two extreme layout philosophies: user-defined layout and automatic layout. In user-defined layout the position and size attributes are completely defined by the editor user. An example is a graph-editor, where the size and position of nodes as well as the routing of line connections have to be specified by the user. When the user constructs a layout that does not meet the constraints of the language, the corresponding modification is rejected. When automatic layout is used, the layout attributes are completely computed from the language structure. The user has no possibility to modify the layout. An example is the layout computation for an ANDSuperstate in the previous section. The size of the ANDSuperstate is computed automatically from the size of its subelements.

In practice, a mixture of these philosophies is used: some layout decisions are made by the user, others are derived by the editor. This is also true for our Statechart example: some properties like the size of an ANDSuperstate or the position of the ANDStateRegion nodes are computed, others like the position of the whole ANDSuperstate can be chosen by the user.

In attribute computations the direction of layout dependencies is fixed: the size of an ANDSuperstate is computed from the size of the ANDStateRegion nodes. It can never be changed directly. To enable undirected layout attribute dependencies graphical constraint solvers were developed. They can propagate layout dependencies in both directions. When the size of an ANDStateRegion is changed, the size of the including ANDSuperstate is adjusted. When the size of the ANDSuperstate is changed, the size of the corresponding ANDStateRegion nodes is affected.

To support this kind of layout strategy, the VL-Generator has an interface to the constraint solver Parcon [8]. There is a data type SolverValue which can be used for attribute values. SolverValue is basically an integer value, which can be updated by the constraint solver. There are also functions that allow one to constitute equalities and inequalities over Solvervalues and to initiate the constraint solver run. The constraint network is specified by attribute computations, which are similar to those presented in Section 2.1.

There are kinds of layout computations (e.g. line routing and graph layout algorithms) which cannot be specified by constraint networks because constraint solvers are usually restricted to linear equalities or inequalities. One can apply such computations by attribute grammars, but one needs knowledge about the line routing or graph layout library used. However, in VL-Eli this knowledge is encapsulated in reusable specification libraries. The language developer does not need to know about interfaces to graph layout, line routing or constraint solver interfaces. All that knowledge is encapsulated in visual patterns, which are presented in the next section. 


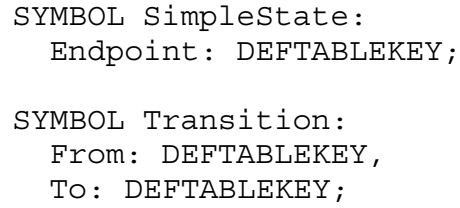

Figure 9. Attribute definitions for cross-relations.

\subsection{Cross-relations and semantic checks}

A tree grammar like that in Figure 4 specifies the hierarchical relation 'consists-of' between language constructs. Almost every language has means to express other relations between arbitrary tree nodes, for example defining and applied occurrences of one program entity. Such relations are usually expressed by identifiers in textual languages and by line connections in visual languages. It is a well-established compiler technique to represent a program entity and its properties by an entry in a definition table. Wherever a tree node refers to a program object an attribute contains a reference to such an entry of the definition table.

VL-Eli adapts this technique to represent any kind of non-tree relations: a specific kind of attributes is provided which contains references to connector objects in the definition table. Two program constructs are considered to be connected if attributes of their nodes refer to the same connection object.

An example is shown in Figure 9. In our Statecharts language a Transition is connected with two states; hence, it has two connector attributes From and To. A Simplestate has one attribute Endpoint. It refers to a connector object which is also referenced by every Transition node connected with that state.

This technique of connector objects does not exclude that semantically wrong connections are constructed, e.g. a Transition may be connected erroneously to another Transition. Computations specified in the attribute grammar check the rules for valid connections and report an error if they are violated.

Figure 10 shows an example for such a computation. The property IsValidEndpoint is defined in all contexts that are valid line endpoints (in this case Simplestate nodes). The value of the property is then checked in the context of Transition nodes to determine whether the connection is valid. The attribute ROOT . endpointsDefined is needed to establish a dependency between the Reset and the Get function calls: all Reset calls must occur before any call of the Get function. If the connection is invalid, the library function message is called with an appropriate message text. All computed messages are collected and displayed in a message window. (In Figure 1 a similar message reports an invalid connection of an initial state.) Selecting one of those messages highlights the representation of the corresponding tree node, which is given by the _currn parameter. Of course, this mechanism is not restricted to line connections, it can be applied to any kind of semantical constraints.

Certain semantical constraints are automatically established when a visual pattern is applied. For example, when a Line pattern is used to visualize Transition nodes, the lines may only connect nodes that inherit the role LineEndpoint. In contrast to the checking described above, 


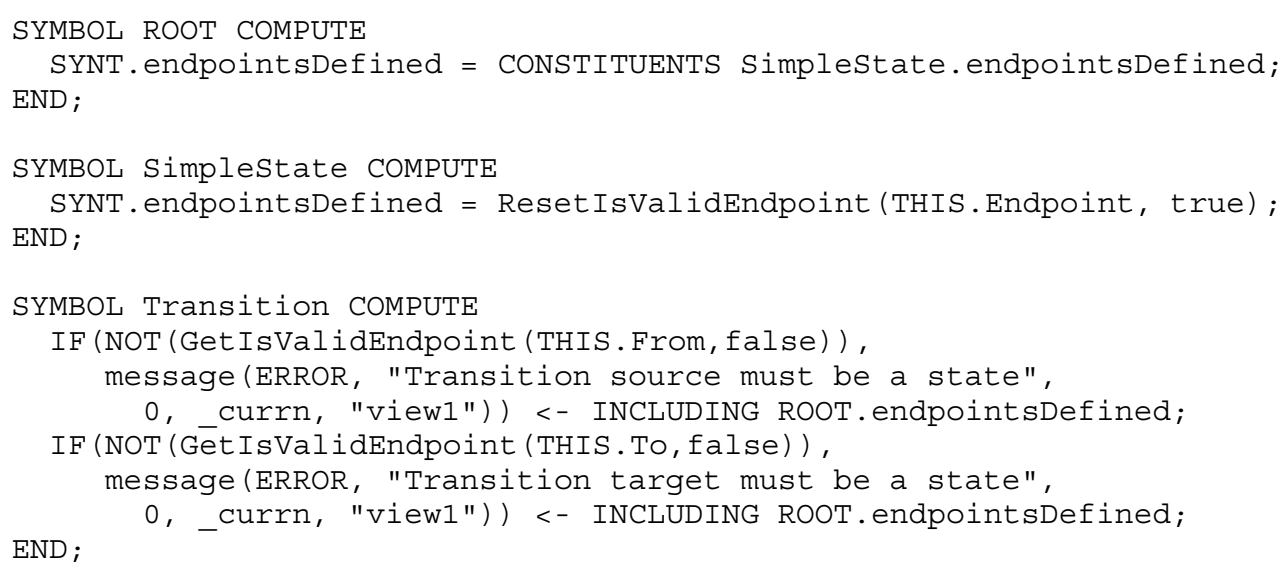

Figure 10. An example for semantical checks.

pattern implementations enforce these constraints. This is straightforward, since one responsibility of visual patterns is the implementation of interactive behavior.

The outlined specification concept allows any kind of cross-relations and any kind of semantical constraints which can be much more complex than just checking types and cardinalities. Additionally, the resulting structure corresponds to the results of name analysis in compiler construction. Thus, the implementation of subsequent analysis and translation is independent of the frontend, regardless whether it is visual or textual.

\section{PATTERN-BASED SPECIFICATIONS}

Visual patterns introduce another level of abstraction on top of the specifications for the VL-Generator. They provide a conceptual relation between the abstract program structure and the structure of visual presentations, as well as a means for precoined specifications of visual language constructs. In this section we describe (1) the concept and implementation of visual patterns and (2) their applicability in a great variety of visual languages.

\subsection{Concept and implementation}

Let us consider the conceptual level first, which is depicted in Figure 11. A visual pattern is used to describe the abstract structure, the interaction operations, and the visual concept of a language construct. For example a XORSuperstate can be described by the Set pattern, which states that it contains an unordered collection of elements, has operations for insertion and deletion, and is visualized by drawing the elements at arbitrary positions, but without overlapping. Choosing the List pattern would violate the intention of the XORSuperstate, because this would imply a certain order 


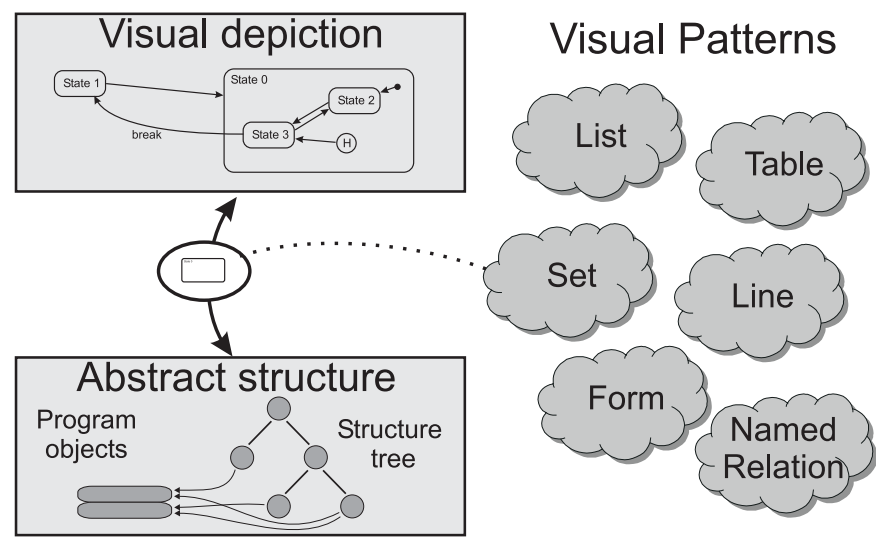

Figure 11. Pattern-based specification.

Set

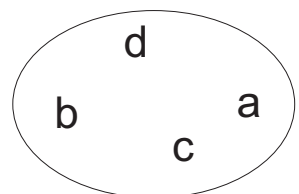

List

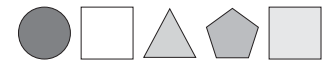

Table

\begin{tabular}{|l|l|}
\hline Conference & \multicolumn{1}{c|}{ Date } \\
\hline AVI2002 & May, 22-24 \\
\hline ETAPS2002 & April, 6-14 \\
\hline
\end{tabular}

Form

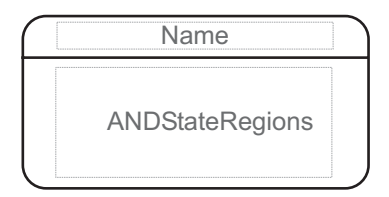

Line

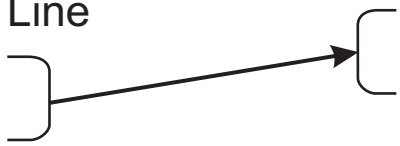

Named Relation

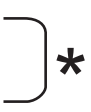

Figure 12. Visual patterns.

of its elements. A designer of a visual language may express the abstract structure and the visualization concepts using the notion of visual patterns.

A selection of six frequently used patterns is shown in Figure 12: Set, List, Table, Form, Line, and NamedRelation. Although these are abstract concepts as described above we visualize each by a concrete example. 
Both the Set and the List abstraction are mapped to list structures in the tree grammar. However, for sets the order of elements is irrelevant, conceptually as well as for the layout. Note that on the level of the visual patterns no assumption is made about the properties and the representation of the elements of the set or list structure. In the examples of Figure 12 some arbitrary representations have been chosen.

The Form pattern maps directly to the tuple given by the symbols on the right-hand side of a single tree production, in the simplest case. For example, the ANDSuperstate consists of two components, its name and its ANDStateRegions. In general the Form pattern allows one to represent a tuple which is built from nodes of the subtree. The tuple elements are marked by the role FormElement.

A Table represents a sequence of tuples which all have the same structure. The tree structure is a list of tuples. The elements of the list inherit the role TableRow and the tuple elements inherit the role TableCell. A similar graphical representation could be achieved by combining the List and the Form pattern. However, the Table abstraction is needed because it captures the relationship between corresponding components of the table-the columns. The combination of List and Form pattern would not align them. Further, the Table pattern provides mechanisms to define a heading for each column.

There are two patterns that conceptually connect components of a program which are represented by tree nodes. The Line pattern captures any kind of line being drawn between the components, whereas the Named Relation pattern represents a relation based on equal names or tokens. The latter concept also allows for links between components in different windows.

All these are abstract concepts, which are characterized by typical graphical properties like 'order along a certain direction' or 'arbitrary position inside a certain region'. The concrete graphical representations of a particular visual concept can be very different.

The notion of visual patterns provides a means of reusable implementation specification. For each visual pattern concrete variants are described in terms of composable specifications for the VL-Generator. Such a pattern variant encapsulates operations that are needed for a visual structure editor to implement a certain graphical representation of the structural abstraction. That is operations which:

- layout the components of the structure, e.g. the elements of a list within the provided screen area;

- draw the graphical representation, e.g. the border of a list together with lines that separate list elements; and

- provide facilities for user interactions, e.g. insert or delete list elements.

These operations are implemented by computations in terms of attribute grammars as described in the previous section. The facilities for user interactions are achieved by integration of context information and event handlers into the data structure for graphical representation. This is supported by the VL-Generator as part of the interface to the Tcl/Tk layer.

The pattern roles are easily adaptable to the needs of the language developer and are flexibly combinable with other computational roles. To cover a wide range of visual representations, we provide mechanisms to parameterize the computations. Of course this means that the implementations of pattern roles are more complex than the implementation of individual solutions.

To demonstrate the structure of a pattern implementation, we present a simplified specification (Figure 13). The purpose of this pair of roles is to compute the representation of a list, its insertion points, and to define the positions of its elements. 


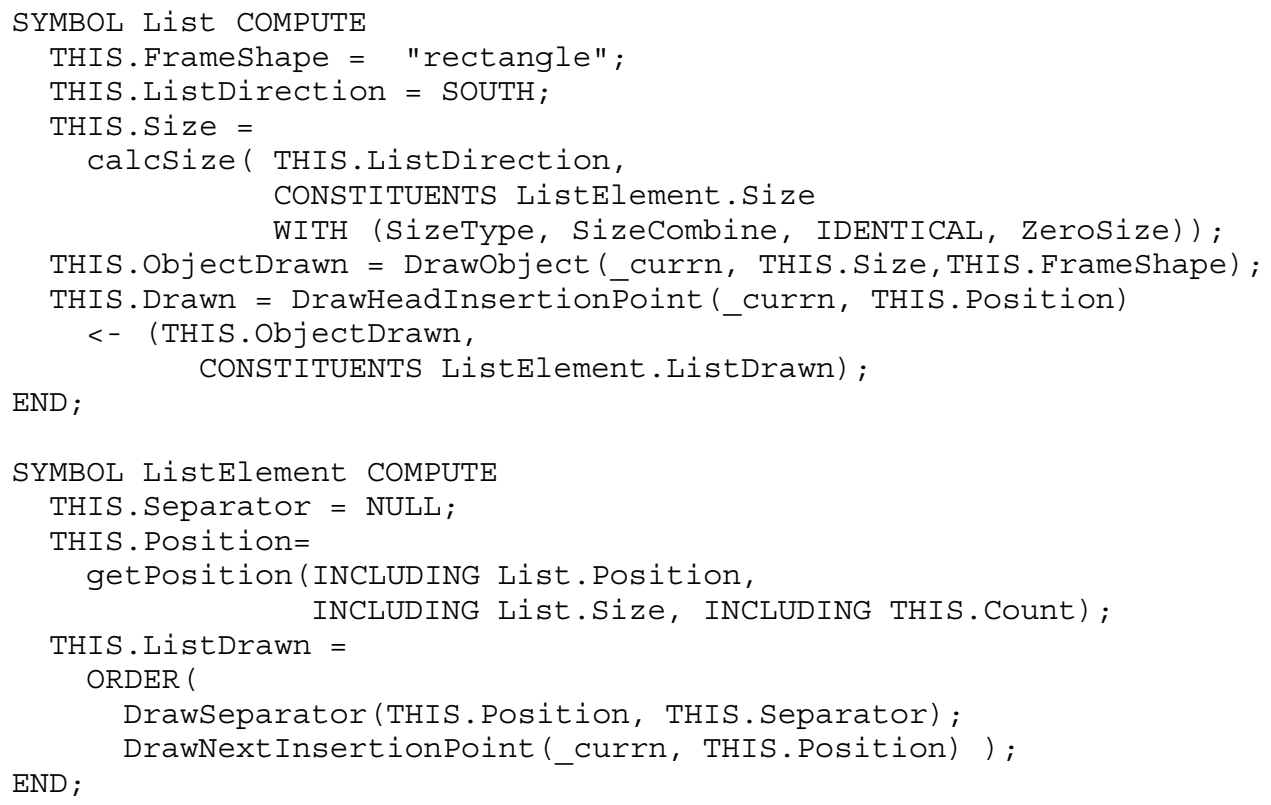

Figure 13. Example of parameterizable pattern implementations.

The computation of the layout depends on some attribute values that have to be defined in other computations: the Si ze attribute of list elements is used to determine the size of the list representation and the Position attribute of the list together with the Size attributes of the list elements are used to determine the position of the list elements. List.Size is a synthesized attribute of List nodes because it depends on the structure of its subtree. List. Position is an inherited attribute of List nodes. It is not computed but only used by the computational role List because it depends on the surrounding visual constructs.

The result of the layout computation is used to create a graphical representation. Each call of an interface function like DrawObject adds a graphical primitive (for example, a shape, a text, or an image) to the visual workspace, gradually defining the complete representation. Conceptually, each computation starts with an empty workspace. Note the argument_currn of this function call. _currn is a reference to the tree node for which this computation is performed. Using this parameter, interactive operations like 'delete' can be traced back to the corresponding node of the tree.

To make the editor more intuitive, the functions DrawHeadInsertionPoint and DrawNextInsertionPoint add additional context information to the graphical workspace. They create so-called insertion points, which are invisible under normal circumstances and located between the list elements. When the user wants to insert a new list element the nearest compatible insertion point is highlighted, indicating the position where it may be inserted. In this way, the user can easily insert a new element by simply dragging it to the desired position on the graphical workspace. 
(Note that the implementation of the interaction mechanisms is also pattern specific because different graphical structures need different interactive operations.)

There are attribute computations (for example, for the Frameshape attribute) that do not depend on other attributes; they are constant. We introduced separate attribute values for these constants to allow them to be overridden. For example, the attribute ListDirection is passed to the calcSize function, defining whether the list layout should be vertical or horizontal. The default value SOUTH can be overridden by the language designer to choose a different layout. For example, to apply the generalized list role to the ANDStateRegions node, which should be drawn horizontally one specifies

SYMBOL ANDStateRegions INHERITS List COMPUTE

THIS. ListDirection=EAST ; END;

The mechanism is sufficient for flexible parameterization of pattern variants. The attributes can have complex types and their values can be constructed by complex computations. The default computation need not yield a constant value but can depend on other attributes. Since the whole implementation is based on attribute computations, all parts can be re-implemented when they are not sufficient for a specific application. In most cases, adequate parameterizability can be achieved by orthogonal attributes with simple types like enumerations, integers or character strings.

Figure 14 shows how visual patterns are combined to implement the Statechart language. The specification contains an application of the List pattern for ANDStateRegions as described before. The representation of ANDSuperstate nodes is defined by the Form pattern. This pattern application substitutes the hand-coded specification in Figure 5 in the previous section. The computation for Simplestate nodes demonstrates how language primitives can be implemented. Several roles encapsulate different kinds of simple graphical objects. In this case the role TextLeaf is used, which implements text that is editable in place. The last pattern application defines the implementation of the Transition nodes. They are represented by lines which connect other language elements. The role EndPoint marks language elements where line endpoints can be attached.

The specification also shows how applications of visual patterns are combined to implement a visual language. For example, the symbol ANDSuperstate inherits the roles Form, SetElement, and EndPoint because it is visualized by a form representation, is an element of a surrounding set of states, and a potential endpoint of a line, i.e. a target or source of a transition.

The parts of the specification not shown in Figure 14 contain similar descriptions for other language elements (i.e. the representation of XORSuperstate, HistoryState, Initialstate, and Finalstate). Additionally, display properties of Form representations (e.g. the value of ANDStateDrawing) and the buttons to insert language elements are specified in other specification languages.

In general, the patterns provide reasonable defaults for almost all variable properties. Hence, acceptable solutions can often be obtained with very little effort, i.e. just by selecting the pattern variant and stating the inheritance relation between grammar symbols and pattern roles. Even if some computations are overridden others are still reused from the pattern.

The described specification technique of symbol roles which provide computations to be inherited by grammar symbols is a characteristic property of our attribute grammar language. In the Eli system 

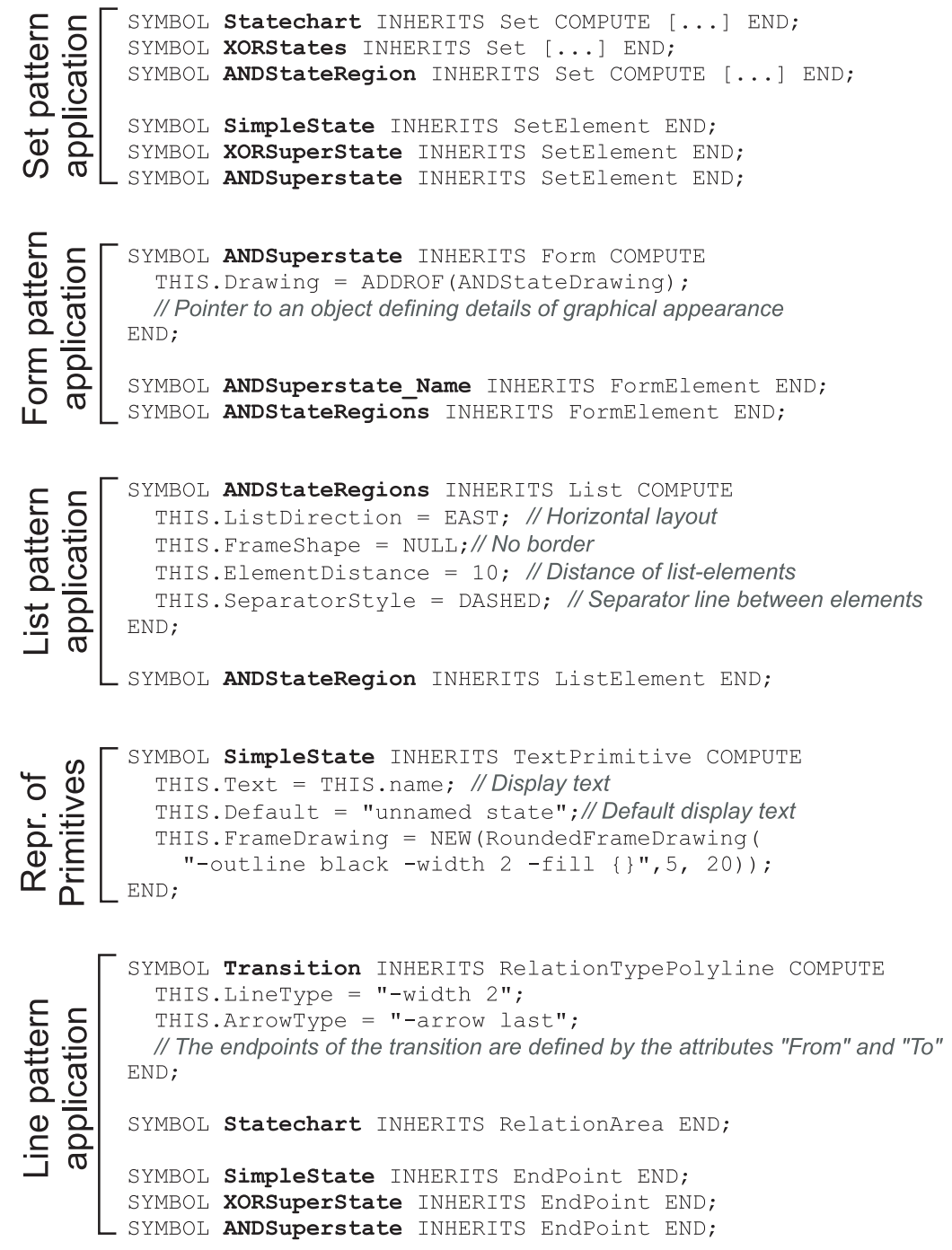

Figure 14. Part of the specification for the Statechart diagram language. 


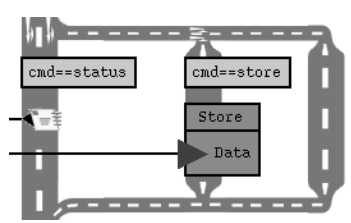

(a)

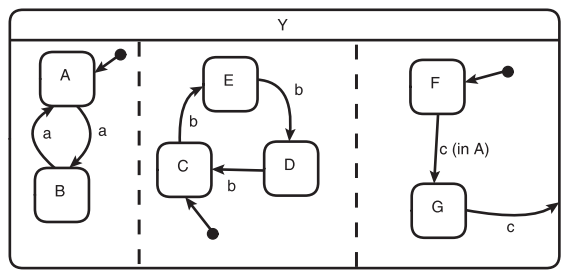

(b)

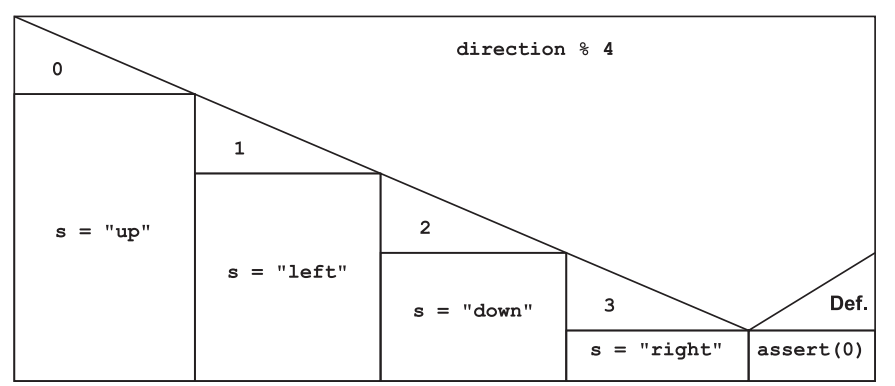

(c)

Figure 15. Instances of the List and FormList pattern: (a) If-Cascade in Streets; (b) and-Superstate in UMLStatecharts; (c) CASE-Construct in NSD.

this technique has proven to be very effective. Precoined solutions for common tasks in the areas of name analysis, type analysis, and transformation are provided by a collection of computational roles, which are stored in generic module libraries [26]. Here we apply that method for common tasks in visual language implementation.

\subsection{Reusability for different languages}

In the following we demonstrate that visual patterns suffice to implement a great variety of visual languages.

Instances of a visual pattern can have very different graphical representations which may need rather different implementation techniques. We demonstrate this for the List pattern which occurs in almost every language. Figure 15 shows three very different visualizations of lists taken from three languages.

Figure 15(a) shows an if-cascade of the language Streets, a visual language for modeling parallel processes [28]. Its central visual paradigm is a road system representing the control flow. The if-cascade is modeled by an arbitrary number of guarded branches and one 'else' branch. In the example, there is one branch with condition $\mathrm{cmd}==\mathrm{stat}$ s, another with condition $\mathrm{cmd}==$ store and an empty 'else' branch. The control flow goes from top to bottom. The branches depart from the main road which is located on the left-hand side. The graphical representation of this language is based on icons which are 
arranged on a grid. When another branch is inserted into the sequence, further street icons are created and placed on the grid to connect the new branch with the existing ones.

The if-cascade is specified by an instance of the FormList pattern (see Table I). It is obtained by applying three forms recursively, one for the root of the sequence, one for the inner elements, and one for the last element with the else branch. The representation of each form is defined by a matrix of images, which contains components of the street. Some rows or columns of the matrices are automatically replicated to obtain a larger road system.

The superstate in Figure 15(b) is taken from our UML Statechart editor. It comprises a sequence of component regions arranged within a box side by side in a row. The regions are separated by dashed lines. The visualization is provided by the List pattern. The pattern application is parameterized to yield a horizontal layout and the dashed separator lines, as described in Section 3.1.

Figure 15(c) is an example of a switch-statement in Nassi-Shneiderman notation [29]. The structure corresponds to a list, where each case $(0,1,2$ and 3$)$ is a list element. There are two additional components: the switch expression (direction $\div 4$ ) and the default case. The particular challenge of the graphical representation is the diagonal layout of the sequence elements.

The representation is obtained by applying the FormList pattern as for Figure 15(a). Again, three forms are applied recursively: one for the root of the sequence, one for the inner elements, and one for the last element with the default case. In contrast to Figure 15(a) the representation of this form is not defined by a matrix of images, but by a collection of vector graphic elements. To enlarge the representation of the switch construct, the coordinates of the graphical elements are transformed automatically.

We thoroughly analyzed eight visual languages: UML class diagrams, UML sequence diagrams, UML Statechart diagrams [1], Nassi-Shneiderman diagrams [29], Query-by-example tables [30], Pictorial Janus [31], LabVIEW [2], and Streets [28]. They can be considered as representatives of rather different visual styles and exhibit a large variety of graphical elements. Each language imposes interesting visual challenges. For example, in class diagrams the source or target of a line connection can again be a line. Sequence diagrams have a remarkable visual structure that requires unusual graphical constraints. Statechart diagrams have a complex structure of nested states. Nassi-Shneiderman diagrams require different styles of list layouts. Query-by-example uses table-like graphical structures. Pictorial Janus uses the 'touches' relation. LabVIEW is an example of languages that are based on orthogonal views showing the same program elements. Streets relies on complex decorations.

As a result of this analysis we defined ten visual patterns and developed implementation variants as shown in Table I. A pattern has a characteristic visual concept and is based on a specific structural abstraction (e.g. a List is a visualization of a sequence, a Form is a visualization of a tuple). Each pattern provides computational roles, which are used to relate them to the tree grammar (column 'Roles'). In the following we outline characteristics of the ten patterns and describe the implementation variants.

The patterns Form, List, Set, Table, Line, and NamedRelation are already explained in Section 3.1 and are visualized in Figure 12. Since they are quite intuitive, we do not repeat their descriptions.

The pattern FormList combines a Form and a List representation. The underlying structure is a tuple, where one tuple element is a sequence. The combination into a single pattern is needed when the 
Table I. Visual patterns and their variants.

\begin{tabular}{|c|c|c|c|c|c|}
\hline Visual pattern & Roles & $\begin{array}{l}\text { Layout } \\
\text { freedom? }\end{array}$ & $\begin{array}{l}\text { Constraint } \\
\text { solver? }\end{array}$ & $\begin{array}{l}\text { Layout } \\
\text { attributes }\end{array}$ & $\begin{array}{l}\text { Representation } \\
\text { attributes }\end{array}$ \\
\hline \multirow[t]{2}{*}{ Form } & Form, & No & No & 1 & 1 \\
\hline & FormElement & Yes & Yes & 1 & 1 \\
\hline \multirow[t]{2}{*}{ List } & List, & No & No & 5 & 4 \\
\hline & ListElement & Yes & Yes & 5 & 4 \\
\hline Set & Set, SetElement & Yes & Yes & 6 & 5 \\
\hline \multirow[t]{2}{*}{ Table } & Table, TableRow, & No & No & 3 & 7 \\
\hline & TableCell & Yes & Yes & 3 & 7 \\
\hline \multirow[t]{2}{*}{ FormList } & FormList, & No & No & 3 & 1 \\
\hline & $\begin{array}{l}\text { ListElement, } \\
\text { FormElement }\end{array}$ & Yes & Yes & 3 & 1 \\
\hline \multirow[t]{2}{*}{ Line } & LineArea, & Yes & No & 6 & 6 \\
\hline & Line, LineEndpoint & No & No & 8 & 2 \\
\hline NamedRelation & NameDef, NameUse & No & No & 0 & 1 \\
\hline Graph & Graph, Node, Edge & Yes & Yes & 6 & 5 \\
\hline RegisterCard & $\begin{array}{l}\text { RegisterCard, } \\
\text { RegisterCardElement }\end{array}$ & No & No & 2 & 2 \\
\hline Stack & Stack, StackElement & No & No & 2 & 1 \\
\hline
\end{tabular}

layouts of both structures are strongly related, i.e. when the list and the form elements are displayed in the same rectangular area (compare Figure 15(c)).

A Graph is a combination of the Set and the Line pattern. The underlying structure is a tuple of two sets: a set of nodes and a set of edges. From the perspective of the Set pattern, the role Graph is a specialization of the role Set and Node is a specialization of SetElement. From the perspective of the Line pattern, the role Graph is a specialization of the role LineArea, Edge is a specialization of Line and Node is a specialization of LineEndpoint. Again, a combination into a single patterns allows one to implement specialized layout mechanisms.

The patterns Stack and RegisterCard visualize a sequence and a tuple, respectively. They have representations that exceed the two-dimensional layout: the elements are 'stacked' in order to save screen space. Only one element is visible at a time and there is a mechanism to switch the currently visible element. This kind of representation is called '2.5-dimensional' [32]. They can be found, for example, in LabVIEW [2].

There are multiple implementation variants for some visual patterns (properties are shown in columns 3-6). An implementation variant encapsulates the creation of graphical elements, layout computations, editor operations, and tree modifications expressed in terms of specifications of the underlying layers. The implementation variants may differ in the layout technique, the graphical characteristics, or in the parameterization model, but they coincide in the pattern abstraction.

Each implementation realizes a concrete layout concept. As we discuss in Section 4.1, there is a trade-off between low layout viscosity (automatic layout) and support for secondary notations (usercustomized layout). Most alternative pattern implementations differ in the degree of layout freedom 


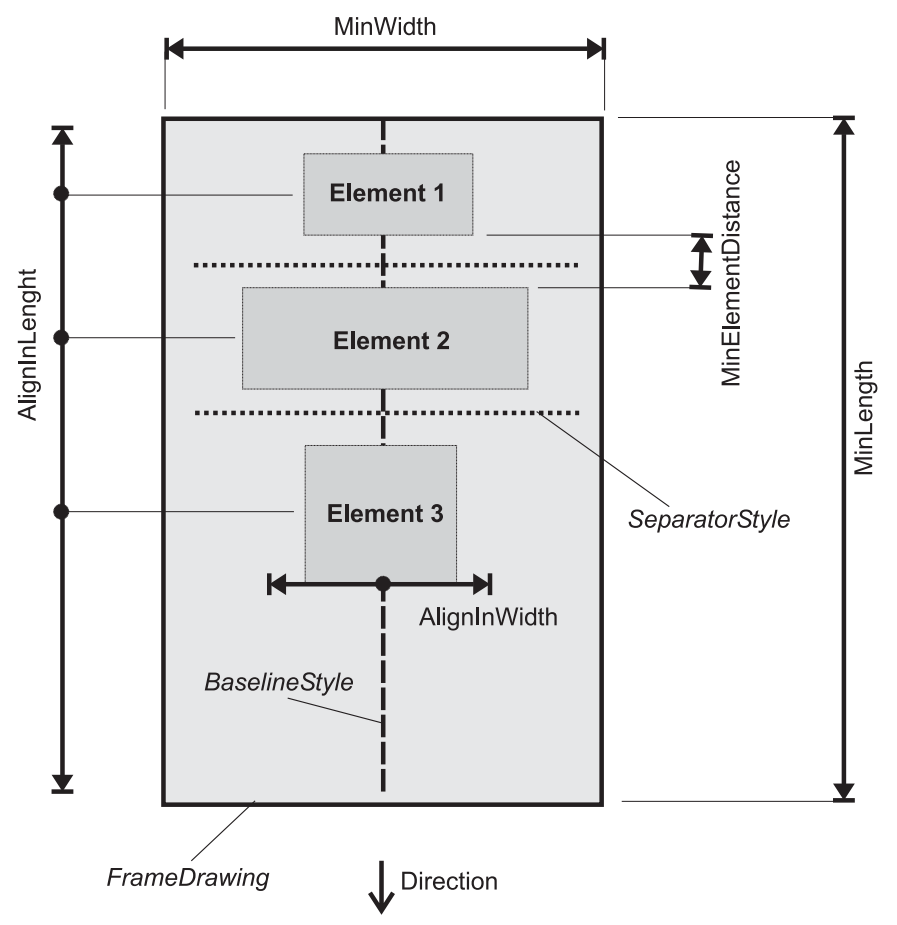

Figure 16. Parametrization options for List pattern implementations.

(Table I, column 3). The choice between the variants depends on the context of the language construct and the desired layout characteristics. Hence, multiple implementations are not necessarily required, but a good choice can improve the user interface quality.

The user-customizable variants (column 3) of the Form, List, Table, and FormList patterns utilize the constraint solver (column 4) to achieve layout freedom. Only one of the Line pattern variants provides user-customizable layout and does not use the constraint solver. It allows manual definition of intermediate points for line routes. One could use a constraint solver to ensure nonoverlapping with other language objects, but the constraints are quite complex and yield results, that are unpredictable for the user.

The second Line pattern variant implements line connections that are routed automatically through routing channels. The variants do not only differ in their layout strategy, but also in their graphical characteristics. The first variant uses (optionally smoothed) polylines (compare Figure 17(c)), the second uses orthogonal lines (compare Figure 17(b)).

Pattern variants can be configured by overwriting dedicated configuration attributes as described in Section 3.1. The configuration attributes of most variants have simple types and orthogonal effects. Two important categories are layout properties (e.g. minimal distance between two list 
elements) and representation properties (e.g. style of a surrounding border). Figure 16 illustrates the configuration model of both List pattern implementations. It shows a list with three list elements and visualizes the effects of important configuration attributes. (Layout attributes are shown in plain font, representation attributes are shown in italics.) The Direction attribute defines the direction in which list elements are lined up. Valid values are North, East, South and West. AlignInWidth defines the alignment of list elements orthogonal to this direction. Valid values are Left, Right, Center, Scale and Buoy. (Scale expands the list elements to the width of the list, Buoy aligns the elements according to alignment points, which are individually defined for list elements.) AlignInLength defines the alignment in list direction. Valid values are Top, Bottom, Center and Scale. (In Figure 16 Top is chosen.) MinWidth, MinLength and MinElementDistance can be overridden to fine-tune the layout. The decorations of list representations can be configured by the attributes Baselinestyle, Separatorstyle and FrameDrawing. Baselinestyle and SeparatorStyle define properties like color, line thickness or hatching of the corresponding decorations. The FrameDrawing attribute stores a so-called generic drawing which is used to define decorations around the actual list. Generic drawings are a powerful specification concept and are specified by a dedicated language.

Most configuration models of other patterns are comparable to the one described above and have a similar number of layout and representation attributes (Table I, columns 5 and 6). One exception is the Form pattern, which is based on generic drawings. Another exception is the NamedRelation pattern, which does not need extensive configuration.

In general, variants of a single pattern may have separate configuration models, but in the case of Form, List, Table, and FormList patterns the corresponding variants share the same model because the implementations differ mainly in layout behavior. In other cases, implementations of the same pattern may offer different parameterization models, e.g. different ones for different kinds of representations or one that is easy to use and one that is more general.

We consider the available variants to be an initial, extensible set which already has proven to be very useful for language implementation. We checked the usability of our approach by implementing five of the analyzed visual languages. In the following we demonstrate how several instances of visual patterns are used to compose these languages (Figure 17).

The Petri-Net language (Figure 17(a)) [33] is an example of languages which are graph-like and require a constraint-based layout. There are two kinds of graph nodes. States are represented by circles and transitions are depicted by filled rectangles. They can be connected by directed arcs, but connections may only go from states to transitions or from transitions to states; a connection between two nodes of the same type is not allowed.

In our Petri-Net specification we use a single application of the Set pattern to lay out the two kinds of nodes, and a single application of the line pattern to define the representation of their connections. The editor does not prohibit connections of nodes of the same type by syntactical constraints. Instead, we specified attribute computations to generate error messages for such constructs.

The implementation of Streets is our most complex example. However, its specification covers the language completely by pattern applications. Figure 17(b) shows a procedure of a Streets process. The control flow is visualized by a system of roads in the right half of the picture. The road contains junctions and icons for operations. The left half contains a set of subprocesses, in this case of type 'worker'. On the left border the procedure's communication interface is defined. It consists of a set of ports which are denoted by triangles. 


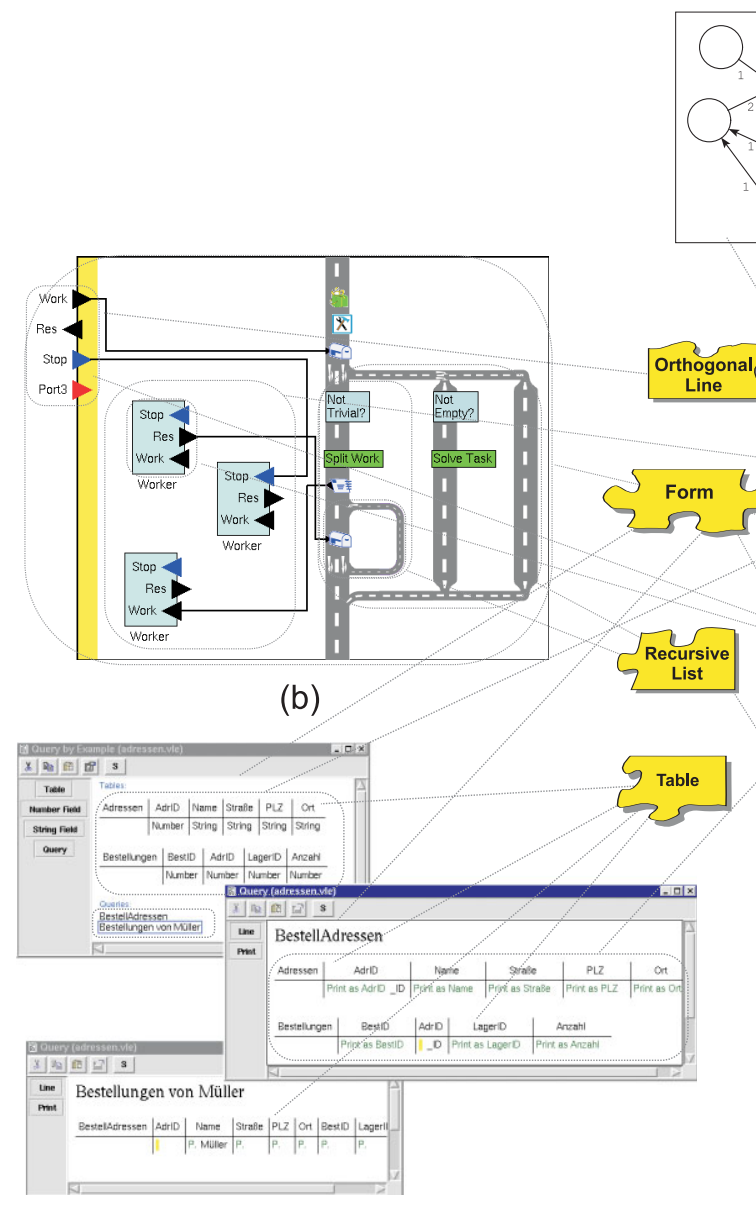

(d)

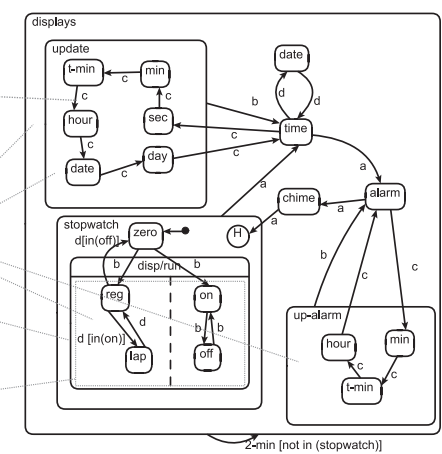

(c)

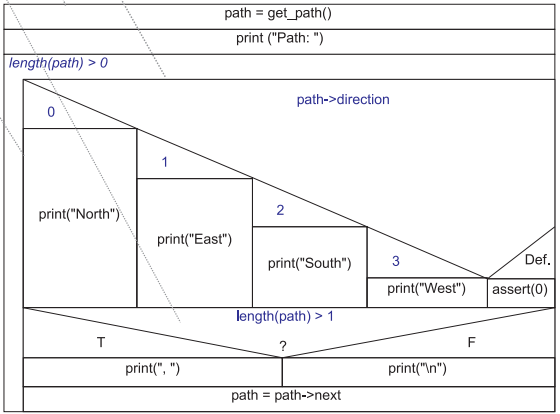

(e)

Figure 17. Visual pattern applications in different languages: (a) Petri-Nets; (b) Streets; (c) Statecharts; (d) Query by example; (e) Nassi-Schneiderman diagrams.

Three instances of the List pattern are used in this representation: (1) the operation sequences on the vertical road segment; (2) the communication ports of the worker threads; and (3) the procedure's communication interface. The if-cascade is produced by a FormList instance as described already in Figure 15. The subprocesses are arranged according the Set pattern. The whole picture is obtained by a Form instance that has three components: (1) the communication interface; (2) the set of worker processes; and (3) the control flow represented by the road system. 
Figure 17(c) shows how visual patterns are applied to implement the Statechart language. It reproduces an example of [34]. The Statechart implementation is mainly based on the Set pattern. It is applied to visualize XORSuperstate, and ANDStateRegions nodes as well as the whole Statechart. The transitions in the diagram are instances of the Line pattern. As already described in Figure 15, the ANDSuperstate 'disp/run' is produced by a List pattern. Since the Set pattern is used, the layout is partially constraint-based. Other parts of the representation, e.g. the size of simple states of AND-Superstates, are computed automatically.

The QBE editor allows the definition of database tables and queries based on the 'Query by Example' method (Figure 17(d)) [30]. The implementation demonstrates the use of tables in visual languages and the realization of structural dependencies between language constructs (in this case table definitions and queries). Figure 17(d) shows an instance that consists of three separate views. The window at the top is the main view. It consists of a list of table definitions and a list of defined queries. These two lists are visually combined by an instance of the Form pattern. Each database table definition is visualized by an instance of the Table pattern.

The window in the middle is a detailed view of a database query. It mainly consists of a list of tables that contain example data defining the query. The structure of the tables is given by the corresponding table definition in the main view. It is a characteristic property of the Table pattern that the structure of the rows is fixed and cannot be altered in a query view. Only complete rows can be inserted in or removed from a table. In this example, all table cells contain simple text elements.

Nassi-Shneiderman diagrams (Figure 17(e)) [29], which are used to represent imperative programs, mainly consist of list structures. Although Nassi-Shneiderman diagrams have low practical relevance today, we implemented this language because it is well known and has some interesting graphical properties.

The sequence of statements is specified by the Simple list pattern variant and the case-construct is specified by the Recursive list variant as described above. We use the Form pattern to implement the if-construct. It has three form elements: the Boolean expression and the two statement lists of the true- and the false-branch.

The QBE as well as the Nassi-Shneiderman example do not use Set, Line or Graph patterns. Thus, the complete layout can be computed automatically. The user does not need (and has no possibility) to adjust the layout.

\section{EXPERIENCE AND EVALUATION}

We have applied our approach and used our tools for the implementation of visual languages with rather different characteristics. They are introduced in Section 3.2. In this section we present the experience gained and evaluate our approach from the view of the language implementor and the language user. We evaluate (1) the quality of the user interface, (2) the response times of the editors, and (3) the effort needed to implement a visual language.

\subsection{Quality of the user interface}

Language specific structure editors—visual as well as textual-have to meet high requirements on their usability because they compete with well-established general purpose editors which users are 
accustomed to. Users will not give up free style drawing and typing unless they really benefit from language-specific guidance and support. Our approach of generating visual structure editors offers the chance to capture high-quality solutions in precoined specification modules based on expert knowhow. However, it is to be shown whether that quality carries over through pattern applications into the generated products for rather arbitrary visual languages.

There is a lot of work in the area of user interfaces in general [35,36] and for user interfaces in language environments in particular [27,37,38]. A good user interface for visual languages must achieve multiple goals.

- The interaction model should be easily understandable. Unexperienced users should be able to get into work quickly. This can be accomplished by applying standards for menu structures and key-bindings as well as using widespread interaction concepts like context menus or methods to create line connections. In general, the interaction model should be as simple and homogeneous as possible.

- Changes should require low mental effort [39]. The user should concentrate on contents rather than user interface interaction. Thus, the user interface has to be 'intuitive'. Minor [38] suggests direct manipulation and a palette-based language element selection in structure editors. Often, mode-less user interfaces are recommended in contrast to mode-driven systems like vi.

- Changes should require low physical effort [39]. The physical effort measures the speed in which a particular operation can be performed by the user. To decrease the physical effort for program construction, shortcuts, pop-up palettes, and efficient duplicate protocols are recommended [37]. In general, the number of required interaction steps and the distance of mouse movements should be minimized. Green and Petre [27] call the effort to perform program changes viscosity. Since a program change requires a layout change as well, automatic layout features can further reduce the viscosity.

- Secondary notations [27] should be well supported. Secondary notations carry extra information, and are independent from the formal semantics. Examples are comments, choices of naming conventions and grouping of related program items. In visual languages secondary notations are often introduced by the layout, for example by grouping nodes of a graph. In general, secondary notations depend on the programmer's intuition and cannot be derived from the language structure automatically. Thus, the layout mechanisms have to offer enough freedom to the user to influence the layout in this respect.

- Navigation, browsing and nonlinear program development should be supported. Program development is a nonlinear process. Programmers do not write programs from the first line to the last nor from top to bottom. They iteratively analyze the code written so far in order to knit in a small piece of new code [27]. To support this, handling of incomplete programs as well as browsing and navigation features are needed.

The design and implementation of the visual patterns support these criteria. Hence, they guide language designers towards desirable styles and techniques.

In the following we discuss facilities of generated editors, which contribute to a good user interface. After describing the main properties of generated editors we discuss the following aspects, which we consider particularly important: 


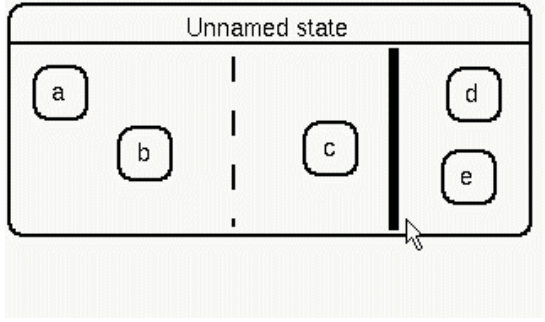

(a)

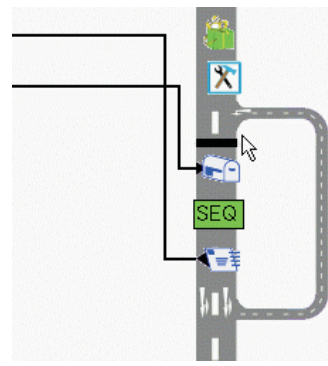

(b)

Figure 18. Editor support of insertions into sequences: (a) Statecharts; (b) Streets.

1. insertion of new structures; and

2. support of graphical layout.

These aspects can be evaluated quite independently of the language design. We give a representative for each aspect and demonstrate how they carry over in different languages.

The overall appearance of editors generated with our tools is shown in Figure 1. The environment meets the standards of graphical user interfaces and has a menu and shortcut buttons for important actions. More than one visual program may be open at the same time and multiple views can be opened for each individual program. In Figure 1 three subwindows show three different views on the same program. Besides the graphical view there is an 'abstract structure tree' view and an 'error message' view.

Subwindows with graphical views have a special toolbar to create new visual objects. In Figure 1 there are buttons for the different kinds of states, ANDStateRegion and Transition nodes.

The quality of interactive operations is most important while creating a visual program. One of the most frequent operations is the insertion of substructures. Let us consider, for example, the insertion of an element into a sequence which has been implemented using one of the sequence related patterns List, FormList, or Stack. Each comes with a powerful insertion mechanism. The points where an element can be inserted are visualized automatically while the mouse cursor passes over the sequence. The user is visually guided, and needs a minimum number of mouse clicks for the operation. Figure 18 shows two editors while highlighting an insertion point of a sequence, ready to be selected with a single mouse-click. The left editor presents a sequence of ANDStateRegion nodes in the Statechart language, the right editor an instruction sequence of the language Streets. This facility carries over into generated products without any contribution of the editor's implementor.

The quality of the layout is mainly determined by the operations and algorithms defining the relative positions of the graphical entities within a larger area of the visual program. In general, the layout decisions can be based on user definitions or can be computed automatically (see Section 2.2). A good layout mechanism should allow easy construction and modification of language expressions without 


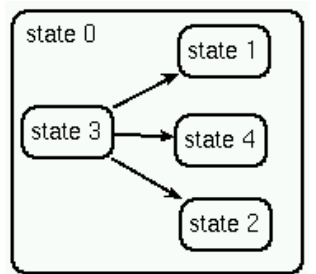

(a)

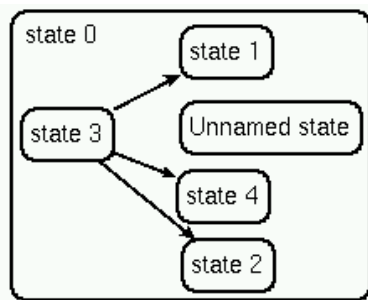

(b)

Figure 19. Layout of states in a XORSuperstate: (a) before insertion; (b) after insertion.

the need for extensive layout adjustments, i.e. ensure low viscosity. However, editor users should be able to adjust the layout to introduce secondary notations.

It is not easy to meet these requirements. Often they seem to contradict one another because it is impossible to decide how to arrange a program after a modification to keep the previously introduced secondary annotations. Which layout mechanism suits best depends on the visual concept and the individual characteristics of the application. Hence, we offer a variety of layout strategies, from which the language designer may select a suitable one for each area of the language.

Figure 19 shows the layout facilities of the Statecharts editor, which is based on constraint networks. The figure shows how the layout is recomputed after inserting a new state. New states can be placed at arbitrary positions, and existing states can be moved as desired, which allows intuitive secondary notations. To ensure a clear and non-ambiguous representation, the constraint solver enforces correct containment and non-overlapping. This means that accidental overlapping of graphical elements is avoided and the necessity of user actions for layout purposes is minimized. For example, one need not make room for the insertion of new items. The constraint solver avoids unnecessary movements of objects which are positioned by hand and tries to keep layout changes as small as possible.

\subsection{Response time}

In the following we consider the response time for edit operations which is an essential factor for the usability of a visual structure editor. Users would hardly tolerate that the tool slows them down when creating or modifying a visual program.

An interactive modification causes recomputation of the layout and update of the graphical workspace. The time needed depends very much on the complexity of the program structure and on the layout mechanism. Table II shows response times we measured for modifications of different languages and program sizes. We used a $450 \mathrm{MHz}$ Pentium III system for this measurement.

The computation respectively the update of the graphical representation is divided into three processing steps (columns 3-5). The column 'Attribute' contains the runtime of the attribute evaluator. The mere tree walk of the attribute evaluator does not contribute significantly to the runtime. The time is basically spent in computing the layout values and the constraint network where required. The column 
Table II. Response times for different applications.

\begin{tabular}{llcccc}
\hline Language & Instance & Attribute (s) & Solver (s) & Canvas (s) & Total (s) \\
\hline Streets & Figure 17(b) & 0.144 & 0.020 & 0.219 & 0.384 \\
& Figure 17(b) & 0.157 & 0.024 & 0.227 & 0.409 \\
NSD & Figure 17(e) & 0.039 & - & 0.082 & 0.121 \\
& 50 loops & 0.136 & - & 0.331 & 0.466 \\
Statecharts & Figure 17 & 0.230 & 0.164 & 0.260 & 0.654 \\
& Figure $17^{\mathrm{b}}$ & 0.139 & 2.679 & 0.145 & 2.963 \\
\hline
\end{tabular}

${ }^{\mathrm{a}}$ The change has not disobeyed the constraints.

$\mathrm{b}$ The change has disobeyed some constraints.

'Solver' measures the time needed to process and solve the constraint network. The column 'Canvas' describes the effort to update the internal data structures of the graphical representation. Note that both the 'Solver' and the 'Canvas' computations are partially based on the script language $t c l$. The last column sums up the three values to the total runtime needed for the graphical update.

The columns 'Attribute' and 'Canvas' show that there are no fundamental efficiency problems. Since the whole representation is re-computed after a change the type of change is not relevant for these values. Even though we use a non-incremental attribute evaluator and a large amount of interpreted code, the recomputation of a Nassi-Shneiderman diagram with 50 nested loops requires only $0.5 \mathrm{~s}$. In practice, more complex diagrams will hardly ever appear, because they will probably be decomposed into individual views, which can be displayed separately.

The response time of the constraint solver is the only critical value. The recomputation of layout values may need an unacceptable amount of time if not all constraints are fulfilled. This effect arises because the generated constraint systems contain disjunctive constraints. The runtime for solving this kind of constraint system is exponential in the worst case. If the solver has to recompute the layout, the measured runtime can vary significantly, even when the applied changes are similar. Thus, the measured value of $2.679 \mathrm{~s}$ should only be considered as a guide. We are going to attack the problem by allowing the solver to generate a suboptimal solution.

\subsection{Implementation effort}

This section evaluates the effort that is needed to implement a visual language using our methods and tools. The language developer defines the grammar, applies and parameterizes visual patterns and specifies additional computations if unusual graphical representations occur. We also quantify the effect of reuse in relation to the complexity of the visual language. We do not evaluate the effort for extension or modification of the pattern implementations because the patterns are part of the tool and that effort is supplied by the tool provider.

The quantitative data in Table III describe properties of the specification from which we generated the visual editors for the five languages Petri-Nets, Statecharts, Nassi-Shneiderman diagrams, Query 
Table III. Complexity of the editor specifications.

\begin{tabular}{lccccc}
\hline Language & Petri-Nets & SC & NSD & QBE & Streets \\
\hline Productions & 10 & 21 & 24 & 27 & 101 \\
Tree symbols & 8 & 15 & 21 & 25 & 88 \\
Sequences & 2 & 5 & 2 & 6 & 11 \\
Persistent semantical attributes & 6 & 13 & 8 & 13 & 51 \\
Persistent attributes/tree symbols & 0.8 & 1.5 & 0.4 & 0.5 & 0.5 \\
& 48 & 167 & 72 & 133 & 487 \\
LOC attribute computations & 0 & 0 & 0 & 154 & 266 \\
LOC structural constraints & 7 & 37 & 83 & 33 & 349 \\
LOC special purpose languages & 55 & 204 & 155 & 320 & 1102 \\
LOC total view specification & 5.5 & 9.7 & 6.5 & 11.8 & 10.9 \\
LOC view specification/number of productions & 15 & 33 & 23 & 36 & 107 \\
Number inherited symbol roles & 21 & 54 & 33 & 53 & 175 \\
Number of local overriding & 0 & 1 & 0 & 1 & 15 \\
Number of new attribute computations & & & & &
\end{tabular}

by Example, and Streets. The three parts of the table characterize the language structure, the visual properties, and the application of visual patterns.

The number of productions indicates how many language constructs are distinguished in the specification because they differ in structure, in semantics, or in their visualization. Hence, it can serve as a measure of the structural complexity of the visual language. Our five languages span a range from the small Petri-Net language with ten productions up to the ten times larger language Streets.

For each tree symbol there is at least one production. Productions coincide in their left-hand side tree symbol if their language constructs may occur in the same contexts. So the difference between the numbers of productions and that of tree symbols expresses the amount of classification of language constructs, in contrast to mostly individual constructs. There are two reasons why most productions of the five languages are singletons without an alternative for the left-hand side symbol. First, repeated constructs are specified using EBNF notation, and thus do not need alternative productions. Second, most language constructs have different visual appearances. To achieve distinct visual representations by inheritance of symbol roles, the grammar symbols must be distinct. The Statecharts language has significantly fewer tree symbols than productions, because it comprises several forms of states under a common nonterminal.

Persistent semantic attributes are used to store values that describe properties of language constructs they are associated with. Examples for such properties are names, visibility modifiers, or references to other constructs. The latter often occur to connect graphic entities by lines. The number of such attributes per grammar symbol characterizes how much information of the language construct is expressed apart from the syntactical structure. The Statecharts language has the largest average of the five languages, because each state and each transition has a name and lines are used to connect any kind of entity. The latter also holds for the Petri-Net language, which has the second most attributes per grammar symbol. 
The second part of the table describes the size of the view specifications by giving the lines of code (LOC) of hand-coded specifications in contrast to those obtained from specification modules. We distinguish three purposes of these specifications.

- The pattern adaption (LOC attribute computations) comprises code to apply specification modules for visual patterns and to adapt their parameters to the specific needs by overriding some attribute computations. For four of the languages this size is closely related to the number of language constructs. Only the Statecharts specification is significantly larger in this category: as it serves as a demonstrator, the specification is further elaborated, i.e. the default settings are more often overridden by more specific values.

- The structural constraints comprise attribute computations which check the consistency between formal parameters in declarations and actual arguments in applications. Only the languages QBE and Streets have constructs that need such checks.

- The third contribution to the view specification captures two purposes: the description of the language-dependent toolbar of the editor and the graphical decorations of language elements. The latter requires a relatively large amount of specification for form-based languages like NassiShneiderman diagrams, and a smaller amount for table-based languages, such as QBE.

The total sizes of the view specifications demonstrate that elaborated editors can be generated with moderate efforts. Only 204 LOC for a small language like Statecharts and 1102 LOC for a language of realistic size are needed. The size relative to the number of productions are fairly close to each other for the more elaborate languages Statecharts, QBE and Streets. Although they differ in the number of language constructs, the relative size of view specification remains constant. Petri-Nets and NassiShneiderman diagrams have a smaller quotient due to the simplicity of their graphical appearance.

The last group of data in Table III quantifies the use of specification modules, the implementations of visual patterns. Each of the five languages is completely covered by module applications. That means all attribute computations that implement the graphical representation are inherited from symbol roles unless they are overridden. The number of inheritances indicates that each tree symbol inherits on the average one to two symbol roles. Statecharts and Petri-Nets symbols inherit more roles because in both cases the endpoints of lines contribute additional role applications. Furthermore, nesting of states causes a state symbol to play multiple roles: it is a set being a set element of an outer set (see Figure 17(c)). The number of overridden computations shows that one to two computations are overridden per inheritance of a role. This number corresponds to the amount of code for pattern adaption shown in the second part of the table. The module roles in question provide between six and 30 computations. Those which are overridden here set defaults and are intended to be overridden.

The last line of Table III shows how few attribute computations are hand coded to achieve specific graphical effects which are not supported by the specification modules, for example visualizations, which depend on the context of the language construct. The small number supports the statement that the editors are almost completely generated from precoined specifications.

We have generated a complete language processor for Statecharts. In addition to the tasks of the graphical frontend the language processor checks semantical constraints and generates an XML representation. The data in Table III cover only the size for the visual editor part. The sizes of the specifications for the additional facilities of the complete Statecharts language processor are shown in Table IV. 
Table IV. Size of the complete Statechart specification.

\begin{tabular}{lr}
\hline Implementation aspect & LOC \\
\hline Abstract structure & 43 \\
Visual editor & 204 \\
Semantic checks & 53 \\
XML generator & 196 \\
\hline
\end{tabular}

\section{RELATED WORK}

Visual languages and their implementation is a wide area of research and development which has a variety of different facets. It is founded on a large body of published knowledge. An overview over the whole field may start from the bibliography [40] and from the conference series [41]. Here we concentrate on those publications which are closely related to the methods and objectives of our approach. First, we briefly address approaches that specify the underlying structure of visual languages. Then, general concepts for specification and computation of graphical representations are considered, like attribute grammars and constraint networks. Finally, we relate our concept of visual patterns to others on a comparable level of specification. Note that the creation of structure editors is essential for our approach. Hence, we do not compare it to systems that are based on free-hand editing and parsing of diagrams like Penguins [21], or that combine free-hand and structure editing like DiaGen II [14].

The underlying structure of visual languages is specified in terms of different notions. Gigas [42] and Loggie [16] use tree grammars, as VL-Eli does. Gigas only allows hierarchical relationships, Loggie extends the tree grammar by additional edges to allow non-hierarchical relationships. Approaches like Progres [15] use graph-grammars to specify language structures. A graph represents a valid language expression if it can be derived by the given set of graph grammar productions. Graph grammar productions also define the structured edit operations. GenGed [13] uses algebraic graph grammars to model the language structure. Structured edit operations are specified by graph rewrite rules. Other approaches like [43] use class diagrams or ER diagrams to define language structures.

Graph grammars are a powerful calculus, which allows one to capture the structural properties of a language and its transformation by a single formalism. Even properties like validity of line connections can be expressed directly by a graph-grammar. However, a graph-grammar specification may have disadvantageous effects on usability: the interactions of a structure editor may be rather complex if they correspond directly to productions of a graph grammar. When a parser checks a program against a graph grammar, it is difficult to give more specific error reports than 'syntax error' in case of violations.

Attribute grammars, by contrast, are a two-level approach: a context-free tree grammar specifies how the structure of a program is represented. Properties of program constructs and relations between them (the static semantics) are represented by attributes of tree nodes and by data structures like definition tables. This two-level approach coincides with the well accepted decomposition into phases of structuring and semantic analysis. It combines a single representation of the underlying structure 
with powerful mechanisms on the second level. Designers often take advantage of specifying a feature on the semantic level rather than on the structural level in order to provide distinguished error messages. In addition 'cut-and-paste' mechanisms for subtrees come almost for free when using tree grammars. Additional specifications for 'cut-and-paste' are only necessary for intelligent handling of crossrelations.

It is a common strategy to model several levels of abstraction by different structural representations. In Progres, two distinct graph grammars are used to model the logical structure ('abstract syntax graph') and the graphical objects with their geometrical relations ('spatial relations graph'), respectively. The correspondence between the structures is defined by a coupling of the graph grammars. In [43] three distinct UML class diagrams are used to define the 'abstract syntax', the 'spatial relationships' and the graphical representation. The relation between these models are defined by OCL constraints. In VL-Eli the implementation of all graphical representations and the analysis and translation modules is based on the same underlying structure.

Systems based on tree grammars or on graphs often integrate an attribute evaluator mechanism, such that structure-related computations can be specified on a high level. For example, in Gigas, Loggie, or DiaGen the graphical representation is computed or updated using attribute computations. The attribute evaluation algorithm of Loggie-which is based on tree grammars and is extended to allow non-hierarchical relations-has been modified to support computations along these edges. Graph-based systems do not need to distinguish between hierarchical and non-hierarchical relations. Our approach is based on an existing, efficient evaluator, where non-hierarchical relations are processed using side-effect computations on data structures which are controlled by attribute dependencies. Our system integrates concepts to encapsulate precoined solutions. The extension of an existing language implementation system also allows one to re-use tools for analysis and transformation tasks beyond structure editing.

In Progres or GenGed properties of the graphical representation are specified using constraint networks. Graphical primitives are associated to the elements of the underlying structure and a constraint network is defined to constitute mathematical relations between layout attributes (e.g. size, position) of these primitives. In contrast to attribute computations, constraint solvers are generally limited to a restricted subset of mathematical formulas. For example, the constraint solver QOCA [44] only allows linear equalities or inequalities. Parcon [8] additionally offers special constraints to model circles. The computation strategy of constraint systems is more flexible than that of attribute computations because it does not impose a fixed evaluation order. However, constraints are restricted to a set of fundamental mathematical operations, whereas attribute computations may use arbitrary functions.

Many graphical properties can be described by constraints elegantly. Thus DiaGen II offers a constraint-based layout interface as substitution for the attribute evaluator interface. VL-Eli has a mechanism to use a constraint solver during the attribute evaluation to utilize the advantages of constraint networks. However, some relevant problems like graph layout or line routing cannot be solved satisfactory in this way. Furthermore, in some cases efficiency problems are reported to be caused by the constraint solver.

Most of the systems mentioned above do not offer a level of specification which aims at both a highlevel specification and a wide area of language characteristics as our visual patterns do. In GenGed there is a predefined extensible library of high-level constraints like 'touches' or 'contains', which encapsulate sets of low-level constraints. In contrast to them visual patterns are much more powerful. They encapsulate not only layout specifications, but also interactive properties. Vivid [45], a system 
for visual manipulation of database contents, offers a predefined set of layout managers, but they are not defined in terms of an underlying specification level.

Costagliola et al. $[18,19]$ introduce a syntactic classification of visual languages. For each category a generic editor is provided such that simple languages that fit into one of the categories can easily be implemented. For more complex languages, editors are constructed by mixing several syntactic categories. Our visual patterns, however, can be combined in a flexible manner to implement simple or sophisticated languages as well.

From the viewpoint of user interface design the 'visual formalisms' of [46] are comparable with our visual patterns. They are introduced to specify and implement graphical user interfaces for applications which need complex structured input data. Visual formalisms offer a set of building blocks, like tables, graphs, plots, panels, maps, and outlines, which can be instantiated, combined, and specialized. They provide dedicated facilities for graphical editing and browsing of the specified structures. Although the specification level is comparable, that approach aims at the construction of graphical user interfaces rather than at visual language design. The implementation uses framework technology rather than generators, as in our approach.

The studies in [47] use notions which are comparable to our visual patterns to classify representations of different visual information, like lists, matrices, spatial maps or trees, in order to evaluate cognitive properties.

\section{CONCLUSIONS}

This article introduced an approach to implement visual languages. The concept of reusable visual patterns enables language implementation on a high specification level with a rather limited knowledge. It also allows experiments in language design and visualization at reasonable costs.

Our approach supports modularity and locality. The specification of a language processor can be decomposed into modules which each solve a specific task. For example, our complete implementation of Statecharts consists of three modules, one for each of the tasks structure editing, validation of semantic constraints, and translation into XML. The parts can be maintained separately, and can be replaced or removed without effecting other parts. In case of larger specifications like that for the Streets language, a further decomposition into sublanguages is advisable. A good modular decomposition has very positive effects on the maintainability. In our approach this facility is provided by the Eli system.

VL-Eli is used to implement a variety of visual languages. Complete specifications of Statechart diagrams, Petri-Net and other languages can be found on the VL-Eli Web site [48]. Most of the graphical properties are specified by reusing precoined implementations of visual patterns as described in Section 3. VL-Eli is also used in an industrial cooperation project to develop a visual language for the specification of SIM Toolkit applications [49]. The variety of implemented languages shows that the method is applicable to a wide range of visual languages.

The separation of the attribute computation levels and the pattern application level leads to great flexibility in language specification. If there is no applicable pattern for an unusual graphical representation, it can be specified by hand-coded attribute computations and combined with other pattern-based specifications. The implementation of Streets (Figure 17(b)) shows that even complex decorations and metaphors can be implemented. The support for multiple views has proven to be an essential aspect for implementation of complex real-world languages. 
Our experience with the concept of visual patterns and with reusable pattern variants is very encouraging. The approach allows us to realize acceptable solutions for common visual languages with rather limited effort. Continuing work on this line seems to be promising. The initial set of variants needs to be completed and used for further visual language applications. In particular, further insights are expected when the approach and the tools are applied by a larger group of users. Finally, the facilities gained by integration with the Eli system need to be further explored. For example, a textual representation of the abstract structure of a visual program can be easily obtained by Eli tools. Furthermore, a language can easily be implemented with two frontends, one for a visual notation and another for a textual one, both using the same backend.

\section{ACKNOWLEDGEMENTS}

We would like to thank the anonymous reviewers for their valuable and constructive suggestions on previous drafts of this paper.

\section{REFERENCES}

1. Object Management Group. OMG Unified Modelling Language Specification. http://www.omg.org/cgi-bin/doc?formal/01-09-67 [2001].

2. Vose GM, Williams G. LabVIEW: Laboratory virtual instrument engineering workbench. BYTE 1986; September:84-92.

3. Jung M, Kastens U, Schindler C, Schmidt C. Visual patterns in the VL-Eli system. Proceedings of the 10th International Conference on Compiler Construction CC'2001, Genova, Italy (Lecture Notes in Computer Science, vol. 2027). Springer: Berlin, 2001; 361-364.

4. Eli Web site. http://ag-kastens.upb.de/eli_home.html.

5. Gray RW, Heuring VP, Levi SP, Sloane AM. Waite WM. Eli: A complete, flexible compiler construction system. Communications of the ACM 1992; 35(2):121-131.

6. Kastens U, Pfahler P, Jung M. The Eli system. Proceedings of the 7th International Conference on Compiler Construction CC'98, Lisbon, March 1998 (Lecture Notes in Computer Science, vol. 1383). Springer, 1998; 294-297.

7. Ousterhout JK. Tcl: An embeddable command language. Proceedings of the USENIX Association Winter 1990 Technical Conference, Washington, DC. USENIX Association: Berkeley, CA, 1990; 133-146. citeseer.nj.nec.com/ousterhout90tcl.html.

8. Griebel $\mathrm{P}$ et al. Integrating a constraint solver into a real-time animation environment. IEEE Symposium on Visual Languages, Boulder, CO, 1996. IEEE Computer Society Press, 1996; 12-19.

9. Jung M. Ein Generator zur Entwicklung visueller Sprachen. Dissertation, Universität Paderborn, November 2000.

10. Kastens U. Attribute grammars as a specification method. Proceedings of the International Summer School on Attribute Grammars, Application and Systems, Prague, 1991 (Lecture Notes in Computer Science, vol. 545). Springer: Berlin, 1991; $16-47$.

11. Schmidt C, Schindler C. Muster-basierte Generierung von Struktur-Editoren für visuelle Sprachen. Master's Thesis, Universität Paderborn, Germany, January 2000.

12. Grant CAM. Visual language editing using a grammar-based visual structure editor. Journal of Visual Languages and Computing 1998; 9:351-374.

13. Bardohl R. GenGed: A generic graphical editor for visual languages based on algebraic graph grammars. IEEE Symposium on Visual Languages, Halifax, Nova Scotia, Canada, September 1998. IEEE Computer Society Press, 1998; 48-55.

14. Köth O, Minas M. Generating diagram editors providing free-hand editing as well as syntax-directed editing. Joint APPLIGRAPH/GETGRATS Workshop on Graph Transformation Systems (GraTra'2000), Technische Universität, Berlin, Germany, 2000. http://www2.informatik.uni-erlangen.de/download/Papers/GraTra2000.ps.gz.

15. Rekers J, Schürr A. A graph based framework for the implementation of visual environments. IEEE Symposium on Visual Languages, Boulder, CO, 1996. IEEE Computer Society Press, 1996; 148-155.

16. Backlund B, Hagsand O, Pherson B. Generation of visual language-oriented design environments. Journal of Visual Languages and Computing 1990; 1(4):333-354.

17. Crimi C et al. Automating visual language generation. IEEE Transactions on Software Engineering 1990; 16(10):11221135. 
18. Costagliola $\mathrm{G}$ et al. A framework of syntactic models for the implementation of visual languages. IEEE Symposium on Visual Languages, Isle of Capri, Italy, 1997. IEEE Computer Society Press, 1997.

19. Costagliola G et al. Supporting hybrid and hierarchical visual language definition. IEEE Symposium on Visual Languages, Tokyo, Japan, 1999. IEEE Computer Society Press, 1999; 236-243.

20. Zhang D-Q, Zhang K. VisPro: A visual language generation toolset. IEEE Symposium on Visual Languages, Halifax, Nova Scotia, 1998. IEEE Computer Society Press, 1998; 195-202.

21. Chok SS, Marriott K. Automatic construction of intelligent diagram editors. Proceedings of the 11th Annual Symposium on User Interface Software and Technology (UIST-98), San Francisco, CA, 1-4 November 1998. ACM Press: New York, 1998; 185-194.

22. Deransart P, Jourdan M, Lorho B. Attribute Grammars: Definitions, Systems and Bibliography (Lecture Notes in Computer Science, vol. 323). Springer: Berlin, 1988.

23. Waite WM, Goos G. Compiler Construction. Springer: New York, 1984.

24. Kastens U. An attribute grammar system in a compiler construction environment. Proceedings of the International Summer School on Attribute Grammars, Application and Systems, Prague, 1991 (Lecture Notes in Computer Science, vol. 545). Springer: Berlin, 1991; 380-400.

25. Kastens U, Waite WM. An abstract data type for name analysis. Acta Informatica 1991; 28:539-558.

26. Kastens U, Waite WM. Modularity and reusabiltiy in attribute grammars. Acta Informatica 1994; 31:601-627.

27. Green TRG, Petre M. Usability analysis of visual programming environments: A 'cognitive dimensions' framework. Journal of Visual Languages and Computing 1996; 7(2):131-174.

28. Kastens U, Jung M. Streets Abschlußbericht. University of Paderborn, Bericht, 1998. http://www.upb.de/cs/ag-kastens/paper/streets.ps.gz [1998].

29. Nassi I, Shneidermann B. Flowchart techniques for structured programming. Proceedings of SIGPLAN'73, 1973; 12-26.

30. Zloof MM. Query-by-example. Proceedings of the AFIPS National Computer Conference, Arlington, VA, May 1975. AFIPS Press: New Jersey, 1975; 431-437.

31. Kahn K, Saraswat VA. Complete visualizations of concurrent programs and their executions. IEEE Workshop on Visual Languages, Skokie, IL, October 1990. IEEE Computer Society Press, 1990.

32. Glinert EP. Towards 'second generation' interactive, graphical programming environments. Proceedings IEEE Computer Society Workshop on Visual Languages, Dallas, TX, 25-27 June 1986. IEEE Computer Society Press, 1986; 61-70.

33. Petri CA. Kommunikation mit Automaten. PhD Thesis, Universität Münster, 1962.

34. Harel D. On visual formalisms. Communications of the ACM 1988; 31(5):514-530.

35. Nielsen J. Usability Engineering. Academic Press: San Diego, CA, 1993.

36. Redmond-Pyle D, Moore A. Graphical User Interface Design and Evaluation: A Practical Process. Prentice-Hall: Englewood Cliffs, NJ, 1995.

37. Citrin W. Requirements for graphical front ends for visual languages. IEEE Symposium on Visual Languages, Bergen, Norway, 1993. IEEE Computer Society, 1993; 142-150. citeseer.nj.nec.com/citrin93requirements.html.

38. Minor S. Interacting with structure-oriented editors. International Journal of Man-Machine Studies 1992; 37(4):399-418.

39. Norman DA. Cognitive engineering. User Centered System Design: New Perspectives on Human-Computer Interaction. Lawrence Erlbaum Associates: Hillsdale, NJ, 1986.

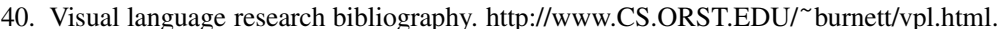

41. IEEE Symposium on Human-Centric Computing Languages and Environments. (Replaces the IEEE Symposium on Visual Languages series.)

42. Franchi-Zannettacci P. Attribute specifications for graphical interface generation. Information Proceedings '89. NorthHolland, 1989; 149-155.

43. Akehurst DH. An OO visual language definition approach supporting multiple views. IEEE Symposium on Visual Languages, Seattle, WA, September 2000. IEEE Computer Society Press, 2000. http://www.cs.ukc.ac.uk/pubs/2000/1097.

44. Marriott K, Chok SS, Finlay A. A tableau based constraint solving toolkit for interactive graphical applications. Technical Report 98/07, School of Computer Science and Software Engineering, Monash University, Australia, June 1998.

45. Dangberg A, Müller W. Generation of interactive visual environments for direct manipulation of database content. IEEE Symposium on Visual Languages, Tokyo, Japan, 1999. IEEE Computer Society Press, 1999; 178-179.

46. Zarmer CL, Chew C. Frameworks for interactive, extensible, information-intensive applications. Proceedings of the ACM Symposium on User Interface Software and Technology (UIST), 15-18 November 1992. ACM Press: New York, 1992; 33-41.

47. McWhirter JD, Nutt GJ. Escalante: An environment for the rapid construction of visual language applications. Proceedings 10th IEEE Symposium on Visual Languages, St Louis, MI, 1994. IEEE Computer Society Press, 1994; 15-22.

48. VL-Eli Web site. http://ag-kastens.upb.de/forschung/vl-eli/.

49. Schmidt C, Pfahler P, Kastens U, Fischer C. SIMtelligence Designer/J: A visual language to specify SIM toolkit applications. Proceedings of the Second Workshop on Domain Specific Visual Languages (OOPSLA 2002), Seattle, WA, 2002. ACM Press: New York, 2002. AG_PAPER_PFAD\#“carstenoopsla2003.pdf”. 